Article

\title{
Space-Time Second-Quantization Effects and the Quantum Origin of Cosmological Constant in Covariant Quantum Gravity
}

\author{
Claudio Cremaschini ${ }^{1, *}$ (D) and Massimo Tessarotto ${ }^{2,3}$ (iD \\ 1 Institute of Physics and Research Center for Theoretical Physics and Astrophysics, Faculty of Philosophy \\ and Science, Silesian University in Opava, Bezručovo nám.13, CZ-74601 Opava, Czech Republic \\ 2 Department of Mathematics and Geosciences, University of Trieste, Via Valerio 12, 34127 Trieste, Italy; \\ maxtextss@gmail.com \\ 3 Institute of Physics, Faculty of Philosophy and Science, Silesian University in Opava, Bezručovo nám.13, \\ CZ-74601 Opava, Czech Republic \\ * Correspondence: claudiocremaschini@gmail.com
}

Received: 26 June 2018; Accepted: 10 July 2018; Published: 16 July 2018

\begin{abstract}
Space-time quantum contributions to the classical Einstein equations of General Relativity are determined. The theoretical background is provided by the non-perturbative theory of manifestly-covariant quantum gravity and the trajectory-based representation of the related quantum wave equation in terms of the Generalized Lagrangian path formalism. To reach the target an extended functional setting is introduced, permitting the treatment of a non-stationary background metric tensor allowed to depend on both space-time coordinates and a suitably-defined invariant proper-time parameter. Based on the Hamiltonian representation of the corresponding quantum hydrodynamic equations occurring in such a context, the quantum-modified Einstein field equations are obtained. As an application, the quantum origin of the cosmological constant is investigated. This is shown to be ascribed to the non-linear Bohm quantum interaction of the gravitational field with itself in vacuum and to depend generally also on the realization of the quantum probability density for the quantum gravitational field tensor. The emerging physical picture predicts a generally non-stationary quantum cosmological constant which originates from fluctuations (i.e., gradients) of vacuum quantum gravitational energy density and is consistent with the existence of quantum massive gravitons.
\end{abstract}

Keywords: covariant quantum gravity; cosmological constant; bohm potential; gaussian solutions

PACS: 03.65.Ca; 03.65.Ta

\section{Introduction}

The theory of manifestly-covariant quantum gravity (CQG-theory) recently proposed in a series of papers (see Refs. [1-6]) provides a possible new self-consistent route to Quantum Gravity and the cosmological interpretation of quantum vacuum. This refers specifically to the quantum prescription of the cosmological constant and the long-standing question whether or not it can be ascribed exclusively to suitable vacuum fluctuations arising at the quantum level.

The crucial feature, that we intend to display in this paper, is in fact that CQG-theory generates self-consistently quantum corrections to the Einstein field equations, i.e., obtained without introducing the semiclassical limit. More specifically, our claim is that CQG-theory actually gives rise to a well-defined quantum prescription of the cosmological constant, its physical interpretation being ascribed to the action of the non-linear quantum vacuum interaction of the gravitational field with 
itself. Remarkably, the new result is based purely on the self-consistent (in the sense indicated above) prescription of quantum vacuum density $\rho_{A}$ obtained in such a framework. In addition it is reached without introducing "ad hoc" phenomenological prescriptions of quantum vacuum, nor possible modifications of the classical Lagrangian formulation of GR based on higher-order classical curvature terms $[7,8]$.

While still not claiming its uniqueness, CQG-theory represents nevertheless a possible new pathway for the establishment of a quantum theory for the standard formulation of General Relativity (GR) and at the same time provides a promising mathematical-physics framework for the investigation of gravitational quantum vacuum effects.

Indeed it is generally agreed that a theory of this type should be, at the same time, in agreement with the fundamental principles of quantum mechanics and quantum field theory [9] as well as the classical Einstein theory of GR [10-13]. The same principles-it should be stressed-notably include the principles of covariance and manifest covariance [14].

CQG-theory realizes, as such, a first-quantization picture of space-time which embodies simultaneously all the required fundamental principles (for an extended related discussion we refer again to Refs. [1-6]). One of its notable features is realized, first of all, by the distinction between a continuum classical background metric tensor $\widehat{g} \equiv\left\{\widehat{g}_{\mu v}\right\}$, yielding the geometric properties of the space-time, and the quantum gravitational field $g_{\mu v}$ which dynamically evolves over $\widehat{g}$ according to a defined quantum wave equation (CQG-wave equation) [4]. The latter is based on the identification of the Hamiltonian structure associated with the classical space-time, with the prescription of the corresponding manifestly covariant Hamilton equations [2] and the related Hamilton-Jacobi theory [3] obtained in the framework of a synchronous variational principle [1]. This leads to the realization in 4-scalar form of the quantum Hamiltonian operator and the CQG-wave equation for the corresponding CQG-state and wave function, whose dynamics is parametrized in terms of an invariant proper-time parameter.

Nevertheless, it must be remarked that relevant results established so far in the framework of CQG-theory pertain both first- and second-quantization effects. The first category includes:

- The establishment of the Schroedinger-like CQG-wave equation in manifest covariant form, which is realized by a first-order PDE with respect to the invariant proper-time [4].

- The statistical interpretation of the CQG-wave equation in terms of corresponding quantum hydrodynamic equations [5].

- The fulfillment of generalized Heisenberg inequalities relating the statistical measurement errors of quantum observables, represented in terms of the standard deviations of the quantum gravitational tensor $g_{\mu \nu}$ and its quantum conjugate momentum operator [5].

- The formulation of a trajectory-based representation of CQG-theory achieved in terms of a covariant Generalized Lagrangian-Path (GLP) approach relying on a suitable statistical representation of Bohmian Lagrangian trajectories $[6,15]$.

- The construction of generally non-stationary analytical solutions for the CQG-wave equation with non-vanishing cosmological constant and exhibiting Gaussian-like probability densities that are non-dispersive in proper-time [6].

- The proof of the existence of an emergent gravity phenomenon occurring in the context of CQG-theory (previously referred to as "second-type emergent-gravity paradigm" (see Ref. [6])) according to which it can/must be possible to represent the mean-field background space-time metric tensor $\widehat{g}$ in terms of a suitable ensemble average. More precisely, as shown in the same reference, this is identified in terms of a statistical average with respect to stochastic fluctuations of the quantum gravitational field $g_{\mu \nu}$, whose quantum-wave dynamics is actually described by means of GLP trajectories [6]. 
Results belonging to second-quantization effects concern instead:

- The proof of existence of a discrete invariant-energy spectrum for stationary solutions of the CQG-wave equation, obtained by implementing the Dirac ladder method (i.e., a second-quantization method) for the stationary wave equation with harmonic Hamiltonian potential [4].

- The analytical estimate for the graviton mass and its quantum discrete invariant energy spectrum, supporting the interpretation of the graviton DeBroglie length as being associated with the quantum ground-state related to the cosmological constant [4]. It must be stressed in this connection that the prediction of massive gravitons represents an intrinsic property of CQG-theory which marks also an important point of distinction with respect to past literature. In fact previous perturbative treatments of quantum gravity based on linearized GR theories typically exhibit-in analogy with the case of the electromagnetic field - massless gravitons. Indeed in the framework of CQG-theory, as discovered in Ref. [4], the existence of massive gravitons and their mass estimate are found to be associated with a non-vanishing cosmological constant.

Based on these outcomes, the target of this paper is to show that in the context of the CQG-theory the equation for the background metric tensor $\widehat{g}$ can actually self-consistently be determined by the CQG-wave equation itself, together with its relationship with the classical Einstein equations. The consequences are of crucial importance since this feature of CQG-theory makes possible the investigation of quantum corrections/contributions to the classical GR equations themselves. In particular, focus is given on the quantum origin of cosmological constant and its quantum representation as predicted by CQG-theory. The consequent second-quantization, i.e., non-linear quantum modifications of the background space-time obtained in this way, represents the main subject of investigation of the present work. As we intend to show, besides quantum gravity theory itself, this is relevant in the context of theoretical astrophysics and cosmology to reach a quantum-gravity interpretation/explanation of selected physical evidences emerging from large-scale phenomenology of the universe.

\subsection{Physical Evidence and Open Problems}

The current status of observations of the large-scale structure of the universe is compatible with its identification in terms of a coordinate-independent (i.e., frame independent) abstract setting realized by a differential manifold $\left\{\mathbf{Q}^{4}, \widehat{g}\right\}$, being $\mathbf{Q}^{4}$ a time-oriented 4-dimensional Riemann space-time with signature $\{+,-,-,-\}$ and $\widehat{g} \equiv\left\{\widehat{g}_{\mu \nu}\right\}$ to be considered a suitably-prescribed background metric tensor characterized by a number of properties.

The first one (EVIDENCE \#1) is about the flatness of the universe [16-19], i.e., the fact that the 4-dimensional space-time curvature is very small and compatible with the existence of a cosmological constant $\Lambda$ which in magnitude is $|\Lambda| \ll 1[20,21]$. However, open questions remain in this regard. These concern, in particular, both the precise physical origin of the cosmological constant [22-25] as well the actual meaning and possible realization of a dynamical evolution of the universe to be realized either in the context of classical or quantum gravity [26-33]. The second physical evidence (EVIDENCE \#2) concerns the apparent lack of large-scale correlations among distant regions of space in opposite directions (i.e., having typical light-ray separation $\gtrsim 10^{9}$ light years) and the consequent occurrence of the phenomenon of (large-scale) homogeneity of the universe, whereby that latter appears to be the same in all directions (isotropic property) [34-43]. Also in this case the physical origin of the phenomenon remains to be fully understood. In fact, it is unclear how distant regions of the universe can undergo or have undergone significant interactions with each other. The third evidence (EVIDENCE \#3) concerns the discovery of an isotropic accelerating expansion of the universe at large distances [44-47]. This implies, in turn, the fundamental consequence that the same cosmological constant $\Lambda$ must be slightly positive in value [48]. The fourth evidence (EVIDENCE \#4) is about the validity of a "Big Bang hypothesis" [49,50], according to which the initial dynamical behavior of the universe should have been characterized by an explosive, i.e., extremely 
fast, expansion/acceleration of space-time starting from the initial condition which in classical GR is understood as an initially-stationary primordial black hole singularity [51-53]. Although several different theories/models have been advanced, the precise physical nature and explanation of the involved phenomena remain still unexplained. Finally, the fifth evidence (EVIDENCE \#5) refers to the conjecture of an inflationary transient phase of the early universe [54-58]. In the original version of the inflationary theory [59] the theoretical inflation model was based on the action of a dynamically-varying scalar field (to be distinguished from the gravitational field) in a local minimum of its potential energy function and rolling the inflation in the primordial era of the universe.

\subsection{Issues about the Cosmological Constant}

Thanks to the evidence provided by astrophysical observations [60], the inclusion of a generic cosmological-constant term $\Lambda$ in the Einstein field equations [61] has nowadays become a well-established part of GR theory. Nevertheless, the cosmological constant $\Lambda$ still emerges, for its possible conceptual implications, as an unsolved issue of outmost importance. According to the literature this can be cast in terms of a decomposition of the form

$$
\Lambda=\Lambda_{\text {bare }}+\Lambda_{Q M}
$$

where respectively $\Lambda_{\text {bare }}>0$ denotes a possible classical contribution and $\Lambda_{Q M}>0$ identifies a quantum contribution. In the present case $\Lambda_{Q M}$ will be identified with $\Lambda_{C Q G}$, namely the contribution arising specifically in the context of CQG-theory.

Regarding the possible realizations of Equation (1), disparate theoretical models have been proposed in the past. These refer both to classical and/or quantum derivations either for $\Lambda_{b a r e}$ or $\Lambda_{Q M}$. In particular, concerning existing models for $\Lambda_{\text {bare }}$ a typical common aspect concerns the adoption of modified classical GR theories. These include for example the so-called Einstein-Cartan gravity theory based on the introduction of torsion effects in the energy-momentum tensor [62,63], the adoption of higher-dimensional space-times (see for example Ref. [64]), Brans-Dicke theories involving the introduction of coordinate-time dependent cosmological constants [65], coordinate-space or coordinate-time varying models [66-70], etc. Regarding, instead, previous theoretical predictions/estimates of $\Lambda_{Q M}$ the list of possible candidates is numerous. A historically famous one inspired by quantum field theory is that $\Lambda_{Q M}$ might be interpreted as due to the quantum vacuum. This involves the conjecture that $\Lambda_{Q M}$ should actually be identified with the total quantum-vacuum energy density arising from all possible quantum fields. In previous literature, estimates of $\Lambda_{Q M}$ based on such a conjecture, i.e., with the inclusion of all the particles corresponding to the standard model and typically a large number of bosonic field components, yield estimates which exceed the experimentally-observed value of $\Lambda$ by nearly 120 orders of magnitude [71]. Therefore, in the literature such a route is usually regarded as to lead to unphysical predictions. It is worth mentioning in this regard that recent numerical calculations including 28 bosonic fields and based on the estimate of the stochastic fluctuations of the associated total quantum-vacuum energy density, rather than the energy density itself, are claimed to provide lower estimates and a resulting acceleration of the universe comparable to the observed one [72,73]. This type of studies should be regarded as complementary to the present quantum theory, although they differ from it for the following main reasons: (1) they are based on numerical calculations, and therefore are subject to the accuracy of the numerical codes actually implemented, while the theory proposed here is analytical; (2) they assume that the source of the universe expansion is the vacuum populated by bosonic fields, while in the present model the cosmological constant is shown to arise purely from quantum gravitational field with its quantum dynamics being predicted by CQG-theory, without needing to invoke any additional field; (3) they realize non-manifestly covariant solutions in which the coordinate time is singled out with respect to space coordinates, while the investigation based on CQG-theory preserves manifest covariance. 
Nevertheless, in part independent of the reasons indicated above, several alternative models have been developed in the literature to explain the expansion/acceleration of the universe as well as the cosmological constant itself (for a review see Ref. [74]). These include, among others: (a) Scalar-field theories based on the introduction of scalar quantum fields and, possibly, related Lagrangian functionals for the variational derivation of the corresponding dynamics $([75,76]$ or the so-called quintessence model $[77,78])$. By comparison, as will be shown below, the present approach differs from these ones in that there is no need to assume "a priori" existence of external quantum fields other than the gravitational one. In other words, the theory proposed here provides a representation for the cosmological constant entering the Einstein equations which is purely generated by quantum interaction of the vacuum gravitational field with itself, independent of the possible additional action of external quantum fields. (b) Perturbative calculations in the framework of loop quantum gravity (see for example Ref. [79]). Here the main difference is provided by the mathematical setting where calculations are performed. In fact, models based on loop quantum gravity approach are intrinsically non-manifestly covariant, in contrast to the present approach which satisfies manifest covariance both at classical and quantum levels (see Refs. [3,4]). In addition, as shown below, the calculation performed in the present framework is not perturbative, but rather it realizes an exact analytical result obtained adopting the trajectory-based representation of CQG-wave equation given in Ref. [6]. (c) Non-commutative approach to the Wheeler-DeWitt equation [80]. Even in this case there are no analogies with the present approach, both because of the non-commutative framework and for the adoption of the Wheeler-DeWitt equation, which is intrinsically non-manifestly covariant in comparison to the CQG-wave equation and also makes use of a Hamiltonian operator derived from preliminary space-time foliation. (d) Theoretical models obtained by introducing quantum corrections in the Raychaudhuri equation [81,82]. Despite referring to Bohmian trajectories, this type of model is phenomenological. In addition, the Raychaudhuri equation is a kinematical equation, not a dynamical one, and therefore inadequate by itself to predict quantum dynamics (see also discussion in Ref. [83]). As a consequence, similarities with the present approach remain excluded also in this case. The CQG-theory in fact is variational, following from preliminary establishment of Lagrangian, Hamiltonian and Hamilton-Jacobi theories for General Relativity, and then implementing a canonical quantization approach. (e) Phenomenological models associated with dark matter and/or corresponding dark energy $[47,84,85]$. Despite being very popular in contemporary literature, dark matter/energy models still lack both experimental evidence and definite theoretical support.

However, despite the huge number of papers appeared so far, no convincing theory or clear physical evidence exists which can explain the physical origin of either $\Lambda_{b a r e}$ or $\Lambda_{Q M}$. In particular, a number of questions regarding the cosmological constant remain. They include in particular:

- ISSUE \#1: the possible quantum origin and, more precisely, the quantum self-generation of the cosmological constant $\Lambda$, i.e., in which the same one is produced merely by the presence of gravitons, as well as its precise estimate in the context of Quantum Gravity.

- ISSUE \#2: the possible dynamical behavior of $\Lambda$ and the search of an admissible dynamical parametrization in terms of physical observables, including the relationship with its constant representation.

- ISSUE \#3: the corresponding eventual implications for cosmology, in particular in reference with the large-scale phenomenology of the universe.

\subsection{Goals and Structure of the Paper}

The problems addressed in the paper are cast in the framework provided by the trajectory-based approach to CQG-theory formulated in Ref. [6] and referred to as generalized Lagrangian-path (GLP) approach. The notable aspect of this representation is that it permits the construction of dynamically-consistent analytic solutions of the CQG-wave equation which lays at the basis of CQG-theory. These include in particular vacuum quantum solutions in cosmological scenarios characterized by Gaussian-like or Gaussian quantum probability density functions (PDF). 
The GLP-approach developed in Ref. [6] refers to the case of stationary background space-time $\left\{\mathbf{Q}^{4}, \widehat{g}\right\}$, i.e., in which the background metric tensor $\widehat{g}$ is considered stationary, namely of the form

$$
\widehat{g}=\widehat{g}(r)
$$

However, the quantum wave-function determined in the same reference exhibits an explicit dependence in terms of the observer's proper time, i.e., a physical observable (see related discussion in Section 3 below). For this reason it is reasonable to conjecture (as shall be shown explicitly in subsequent Sections 6-9) that via second quantization effects, also the same background tensor field might be expected to include an analogous type of dependence. Therefore, the preliminary goal to be pursued as a first task of the paper consists in the appropriate generalization of the theory to a generally non-stationary metric tensor $\widehat{g}$ of the type

$$
\widehat{g}_{\mu \nu}=\widehat{g}_{\mu \nu}(r, s),
$$

where $s$ denotes a suitably-prescribed invariant proper-time parameter. The two settings (2) and (3) will be referred to here respectively as stationary and non-stationary backgrounds. More precisely, for this purpose the role of proper-time and its definition as physical observable are first discussed in the context of both covariant classical gravity (CCG) and covariant quantum gravity (CQG) theories. Then, the extension to the case of a non-stationary background metric tensor is ascertained both for CCG and CQG theories as well as for the GLP-approach presented in Ref. [6]. As a consequence, the non-stationary quantum solutions of the CQG-quantum wave equation determined in Ref. [6] are shown to hold also in such a case.

The second task of the paper concerns, instead, the investigation of the possible validity of the so-called "first-type emergent-gravity paradigm" (see Ref. [6]). Accordingly, the functional form of the Einstein GR field equations should be preserved when quantum corrections implied by CQG-theory and the GLP-approach are retained, consistent with the so-called emergent gravity picture. In other words, the determination of the Einstein equations with quantum contributions included should not depend on the evaluation of semiclassical continuum limit (namely obtained letting in particular $\hbar \rightarrow 0$; see for example Ref. [86] where the derivation of the Einstein field equations was discussed in the context of loop quantum gravity) nor on the prescription of suitable stochastic/quantum expectation values, but rather should be implied by the quantum-wave equation itself. This task should involve the determination of the PDE for the background field tensor $\widehat{g}_{\mu v}$ with inclusion of second-quantization effects arising from the quantum gravitational field itself, to be associated with the corresponding covariant quantum gravity wave equation, i.e., the CQG-wave equation for the quantum state $\psi(g, r, s)$ pointed out in Refs. [4,5]. According to this procedure the CQG-wave equation should deliver the so-called quantum-modified Einstein field equations. These are expected to have the same functional form of the classical equations (see Equation (5) below) but to retain at the same time also well-definite quantum expectation values for the relevant continuum fields, and in particular a quantum expectation value of the cosmological constant $\Lambda$. To carry out this task a number of steps are needed. First, the CQG-wave equation must be shown to imply the validity of a set of Hamilton equations holding for suitable quantum canonical tensor fields and denoted as quantum Hamilton equations. For this purpose the equivalent set of quantum hydrodynamic equations, represented respectively by the continuity and quantum Hamilton-Jacobi equations, are first recalled. Second, by suitably prescribing the initial conditions, the same quantum Hamilton equations must be proved to imply the validity of the quantum-modified Einstein field equations. Third, by explicitly taking into account the quantum solutions determined via the GLP-approach, the analytic expression of the quantum cosmological constant $\Lambda$ needs to be evaluated, with particular reference to its possible explicit dependence in terms of the proper-time $s$ and its consequent identification as a dynamically-evolving cosmological scalar field. Fourth, the solution of the quantum-modified Einstein field equations must be investigated and shown to take the general form of a non-stationary 
background metric tensor of the type given by (3), where explicit proper-time dependences are clearly identified as arising from quantum gravitational contributions. Finally, the asymptotic behavior of the cosmological constant needs to be analyzed in order to understand the physical role of the quantum corrections to the gravitational field cosmological dynamics.

The implementation of this work-plan permits the establishment of a relevant theoretical result, which concerns the investigation of the quantum origin of the cosmological constant. In fact it is proved that CQG-theory predicts quantum-modified Einstein equations which contain a cosmological constant term purely generated by quantum interaction. More precisely, the quantum cosmological constant is shown to arise from the quantum interaction of the gravitational field with itself in vacuum. From the point of view of mathematical treatment, this type of interaction is expressed by the quantum Bohm potential term that is contained in the quantum-wave equation and is made explicit after adoption of the Madelung representation for the quantum wave function and the representation of the same equation in terms of quantum hydrodynamic equations. A characteristic feature of the Bohm potential is that of carrying a non-linear interaction expressed by quadratic first-order derivatives and second-order derivatives of the quantum probability density of the quantum gravitational field tensor. For this reason, the Bohm potential depends also on the explicit realization of the same quantum probability density, a feature which requires the simultaneous solution of both continuity and quantum Hamilton-Jacobi equations equivalent to the quantum-wave equation. The emerging physical picture predicts a generally non-stationary quantum cosmological constant which originates from fluctuations (i.e., gradients) of vacuum quantum gravitational energy density and is consistent with the existence of quantum massive gravitons (see also related discussion in Section 9).

Given these premises, the structure of the paper is as follows. In Section 2, the extension of the functional setting of CQG-theory is presented as appropriate to the treatment of a non-stationary background metric tensor. In Section 3 the role of proper-time in covariant classical/quantum gravity is investigated. In Section 4 the formulation is presented of covariant classical/quantum gravity in the framework of the extended functional setting. In Section 5, the quantum Hamilton equations associated with the corresponding set of quantum hydrodynamics equations are presented. These lead in Section 6 to the construction of the corresponding quantum modified Einstein field equations. In Section 7 the extension is considered of the Generalized Lagrangian Path approach earlier formulated appropriate for the treatment of the extended functional setting. In Section 8 the explicit evaluation of the Bohm effective potential and corresponding source term, together with the identification of the cosmological constant are presented. In Section 9 the proper-time behavior of the quantum cosmological constant $\Lambda_{C Q G}(s)$ and related physical implications are discussed. Finally in Section 10 concluding remarks are pointed out, while completing details of algebraic calculations are reported in Appendixes A-C.

\section{Extended Functional Setting for CQG-Theory}

The theoretical framework of the present paper is couched on the manifestly-covariant Hamiltonian approach for massive gravitons recently developed in Refs. [1-6]. In its classical formulation, referred to as covariant classical theory of gravity (CCG-theory), this is based on a classical Hamiltonian representation for the Einstein field equations of the gravitational field which permits to recover the same Einstein equations as particular solutions of a suitable set of manifestly-covariant continuum Hamilton equations.

The corresponding quantization approach for the space-time metric tensor, denoted as $g$-quantization [5], realizes instead a manifestly-covariant quantum gravity theory (CQG-theory). The characteristic property of CQG-theory is that of yielding a non-perturbative hyperbolic quantum wave equation, denoted as CQG-wave equation, advancing the state of the quantum gravitational field with respect to an invariant proper-time parameter $s$. In addition, both CCG-theory and CQG-theory are manifestly covariant. In accordance, it follows that all the classical and quantum Hamiltonian densities and operators as well as the corresponding continuum coordinates and conjugate momenta, 
are all required to transform as 4-tensors, i.e., to fulfill as such well-definite covariance tensor transformation laws

$$
r \equiv\left\{r^{\mu}\right\} \rightarrow r^{\prime} \equiv\left\{r^{\prime \mu}\right\}=r^{\prime}(r)
$$

associated with local point transformations between reference systems (LPT group [13]), namely in which the rhs of the previous equation depends on the local value of the initial and transformed 4-positions $r \equiv\left\{r^{\mu}\right\}$ and $r^{\prime} \equiv\left\{r^{\prime \mu}\right\}$ respectively.

However, in order that the principle of manifest covariance can actually apply, a background space-time picture must hold. This means that, consistent with experimental evidence, the universe must be identified with a suitable classical curved space-time $\left\{\mathbf{Q}^{4}, \widehat{g}\right\}$ with the background metric tensor $\widehat{g} \equiv\left\{\widehat{g}_{\mu \nu}\right\}$ to be considered a classical tensor field. In particular, this means that the LPT group must leave invariant the differential manifold structure of a prescribed (but in principle arbitrary) curved space-time $\left\{\mathbf{Q}^{4}, \widehat{g}\right\}$, to be referred to as background space-time. Hence, no preferred GR reference frames or coordinate systems are required. The latter occurrence follows for example when decompositions or foliations of space-time (like the $3+1$ representation) and the consequent adoption of non-tensor Lagrangian/Hamiltonian variables are implemented. This typically involves the singling out of the coordinate time to prescribe the dynamical evolution of metric tensor hypersurfaces (see Refs. [87-91]). It must be stressed that although a manifestly-covariant theory of this type needs not necessarily to be unique, the involved notion of manifest covariance given above is certainly unambiguously determined when the background space-time $\left\{\mathbf{Q}^{4}, \widehat{g}\right\}$ is prescribed.

A crucial aspect is therefore the prescription of its functional setting.

One notices in this regard that by assumption $\widehat{g}$ determines the geometric properties of the same space-time and is required to satisfy suitable physical prescriptions. The first one is that $\widehat{g}$ must be considered as a deterministic, i.e., classical, tensor field. As such, in the framework of CQG-theory this is assumed to realize a particular solution of the Einstein field equations. In standard notation the latter can be written

$$
\widehat{R}_{\mu \nu}-\frac{1}{2}[\widehat{R}-2 \Lambda] \widehat{g}_{\mu \nu}=\kappa \widehat{T}_{\mu \nu}
$$

with $\kappa$ being the universal constant

$$
\kappa \equiv \frac{8 \pi G}{c^{4}}
$$

and where

$$
\widehat{G}_{\mu v} \equiv \widehat{R}_{\mu v}-\frac{1}{2} \widehat{R} \widehat{g}_{\mu \nu}
$$

is the Einstein field tensor. Moreover: (1) $\widehat{R}_{\mu v} \equiv R_{\mu v}(\widehat{g}), \widehat{R} \equiv R(\widehat{g}) \equiv \widehat{g}^{\alpha \beta} \widehat{R}_{\alpha \beta}$ and $\widehat{T}_{\mu v}=T_{\mu v}(\widehat{g})$ identify respectively the Ricci tensor, the Ricci 4-scalar and stress-energy tensor (or energy-momentum tensor of matter) all evaluated in terms of the background metric tensor $\widehat{g}$; (2) $\Lambda$ is the still to be determined cosmological constant which can always be taken of the general form (1). Accordingly, the metric tensor $\widehat{g}_{\mu v}$ must raise and lower tensor indices of arbitrary tensor fields, such as for example the second-order coordinate and momentum tensor fields $H_{\mu \nu}=g_{\mu v}, \pi_{\mu v}$, i.e.,

$$
H_{\mu \nu}=\widehat{g}_{\mu \alpha} \widehat{g}_{\nu \beta} H^{\alpha \beta}
$$

with $H_{\mu v}$ and $H^{\alpha \beta}$ denoting respectively corresponding covariant and counter-variant components. The second prescription is that $\widehat{g}$ should determine the Riemann distance on the space-time $\left\{\mathbf{Q}^{4}, \widehat{g}\right\}$ and consequently the proper-time $s$ by means of the 4 -scalar equation

$$
d s^{2}=\widehat{g}_{\mu \nu} d r^{\mu} d r^{v}
$$


Here $d s$ is the so-called line element (arc length) and $d r^{\mu}$ the corresponding 4-tensor displacement around a 4-position $r \equiv\left\{r^{\mu}\right\}$ which belongs to the subset of $\left\{\mathbf{Q}^{4}, \widehat{g}\right\}$ where $\widehat{g}_{\mu \nu} d r^{\mu} d r^{\nu} \geq 0$. As a consequence it follows by integration that

$$
s-s_{1}=\int_{r_{1}}^{r} \sqrt{\widehat{g}_{\mu \nu} d r^{\mu} d r^{v}},
$$

where here $r \equiv r(s)$ and $r_{1} \equiv r\left(s_{1}\right)$ denote two 4-positions along an arbitrary curve (worldline) $r(s)$ joining them (which therefore belong to the same light cone), while $s$ and $s_{1}$ are the corresponding proper-times. In particular, in accordance to Ref. [4], the worldlines on which the Riemann distance is evaluated can be conveniently identified with appropriate non-null field geodetics. Hence, for an arbitrary GR-frame endowed with a 4-position $r^{\mu}$, such a worldline can in principle be identified with one of the (infinite possible) curves that cross the same position, i.e., an arbitrary observer's geodetics $r(s) \equiv\left\{r^{\mu}(s)\right\}$ prescribed in such a way that at proper-time $s$ it coincides with the observer's position, namely so that it satisfies the initial (crossing) condition

$$
r^{\mu}=r^{\mu}(s) .
$$

Here $s>0$ denotes the arc length which is associated with the same observer and therefore is referred to here as observer proper-time. As discussed below (see following Sections 3.1 and 3.2), under suitable assumptions $s$ can be interpreted as a classical 4-scalar observable which can be unambiguously associated with an arbitrary GR-frame. However, the prescription of the proper-time $s$ achieved in this way can also be made unique for all observers thus yielding also a global observable. The third requisite, in close analogy with the quantum wave-function determined in Ref. [6] and for consistency with the goals of the present investigation, is that the background metric tensor $\widehat{g} \equiv\left\{\widehat{g}_{\mu v}\right\}$ should be allowed for greater generality to be non-stationary too. In the context of a manifestly-covariant description, nevertheless, $\widehat{g}$ cannot depend on a coordinate time but necessarily on an invariant time coordinate, to be identified with the proper-time $s$. Therefore, the metric tensor $\widehat{g}$ should conveniently be allowed for greater generality to take the non-stationary form (3).

Regarding classical GR the possibility of an extended functional setting of this type has been already pointed in Ref. [3] as being due either to the action of suitable non-local point transformations acting on GR-frames [13] or to possible non-local source terms in the stress-energy tensor of the Einstein equations. An example of the second type (for the explicit proper-time dependence) arises in particular in the case of electromagnetic radiation-reaction phenomena affecting the dynamics of $N$-body systems of charged particles, with $N \geq 1$ [92,93], where the corresponding stress-energy tensor depends explicitly on the proper-time of the particles subject to radiation-reaction.

In previous works dealing with CQG-theory the case of stationary background metric tensor was actually treated, for which identically $\widehat{g}_{\mu \nu}=\widehat{g}_{\mu v}(r)$. In the present context, however, requiring validity of Equation (3) poses two crucial questions. The first one is whether a consistent generalization of the theory of covariant quantum gravity and of the related GLP-theory developed in Ref. [6] can actually be achieved for a non-stationary background metric tensor of the type (3). The issue concerns also the corresponding formulation of CCG-theory and in particular how the extended and reduced-dimensional variational Hamiltonian structures $\{H, x\}$ determined in Refs. [3,4] can be preserved under assumption (3). The second question instead is about the possibility of predicting the $s$-dependence of $\widehat{g}_{\mu \nu}$ as arising specifically because of second-quantization effects of the gravitational field, namely quantum modifications of the background metric tensor $\widehat{g}_{\mu v}$ and corresponding field equations due to non-linear dynamical interaction of its quantum counterpart field $g_{\mu v}$. In fact, as shown in Ref. [4] the occurrence of an explicit proper-time dependence in the quantum wave-function is a characteristic feature of CQG-theory for the quantum gravitational field, i.e., which arises in the actual construction of particular solutions of the relevant quantum-wave equation of CQG-theory 
based on GLP-parametrization (see also Ref. [6]). As shown in this work, the answer to these questions is deeply related with the investigation of Issues \#1-\#3 posed above.

\section{The Role of Proper-Time in Covariant Classical/Quantum Gravity}

In the context of both CCG- and CQG-theories a crucial aspect concerns the treatment of gravitons, i.e., the quanta of the gravitational field and in particular the related prescription of the notion of proper-time (s). For this purpose one first notices that both in CCG- and CQG-theories the background space-time $\widehat{g}_{\mu v}$ prescribing the coordinate and the geometric properties of the reference system is not quantized. The quantization pertains the fluctuations with respect to $\widehat{g}_{\mu \nu}$ of the quantum gravitational field described by $g_{\mu v}$. The implication is that gravitons still need to be treated as classical particles, i.e., necessarily as point-like neutral, spin-2 collisionless particles, since in order to quantize them one should actually perform a full quantization of the metric tensor defining the space-time, and therefore the physical coordinates identified with position and velocity. In addition, in view of the invariant discrete energy spectrum discovered in Ref. [4] gravitons must carry a non vanishing mass. As a consequence their positions and velocities are considered as deterministic. Therefore they are endowed with a purely geodesic motion while their admissible (or virtual) worldlines must be identified with (deterministic) non-null subluminal geodetics which are associated with the background metric tensor $\widehat{g}$.

Concerning the notion of proper-time, as recalled above, this can be identified with the arc length of a non-null geodetics, i.e., the virtual worldline of a graviton. Such geodetics are intrinsically non-unique. In fact for an arbitrary observer (or GR-frame) defined by its 4-position $r \equiv\left\{r^{\mu}\right\}$, there are infinite geodesic curves $r(s) \equiv\left\{r^{\mu}(s)\right\}$ fulfilling the crossing condition (11) i.e., belonging to the same observer. However, the notion of (observer) proper-time (s) makes sense only if $s$ is an observable. Therefore there must exist a suitable way to prescribe it. In this regard two choices are possible. According to the first case, proper-time is an observer proper-time, i.e., a local observable which may have nevertheless different realizations for each observer (i.e., GR-frames which are mutually connected via the LPT group). In this case the proper-time $s$ is by construction the same one for all geodesic trajectories which cross simultaneously the observer 4-position (see Equation (11)).

The second possible realization is provided instead by the notion of global proper-time, i.e., a global observable which is the same one also for a family of observers which are properly "synchronized" with each other in such a way that the observer proper-time $s$ indeed coincides for all of them. In this case the observer proper-time $s$ takes therefore-by suitable construction-the same value for all such observers. The two choices proposed here require in turn well-definite prescriptions for the functional setting of the observers' geodesic curves. We consider them below.

\subsection{Proper-Time as a Local or Global Observable}

In the first case one can show that a non-trivial definition of the observer proper-time requires that:

(1) For each observer, consistent with its identification with a graviton's virtual worldline, the corresponding geodesic curves (observer geodetics) must be all non-vanishing and oriented (each one with its proper orientation).

(2) Because classical geodetics cannot cross event-horizons of arbitrary black holes (just as classical particles with finite mass), curves originating near them must have a origin point $r\left(s_{0}\right)=r_{o}$ suitably close to the same event horizons. It is understood that the indicated origin point $r\left(s_{0}\right)=r_{o}$ corresponds to a creation point of a graviton's virtual worldline, i.e., a point where a graviton may be created. Hence it makes sense to assume that all the observer's geodesic curves have proper origin points and hence are semi-infinite. In addition, the origin points of all observer geodesic curves cannot coincide with event horizons but can be also arbitrarily close to them, so that the limit of a suitable sequence of origin points actually may coincide with the same event horizon.

(3) For all semi-infinite geodesic curves it makes sense to require that the initial proper-time $s_{o}$ is positive or null. For the uniqueness of $s$ for a given observer-i.e., as a local observable- there must 
exist among all the observer's geodesic curves a (possibly non-unique) observer's maximal geodetics, i.e., a geodesic curve with origin point $r^{\mu}\left(s_{0}\right)$ having the maximal arc length $s-s_{0}$ and subject to the condition

$$
s_{0}=0,
$$

with $r^{\mu}\left(s_{0}\right)$ coinciding (or being suitably close) to the Big Bang event $r^{\mu}\left(s_{0}\right) \equiv r^{\mu}\left(s_{o}=0\right)$, with $s_{0}=0$ to be referred to as Big Bang proper-time.

When interpreted in a cosmological scenario, such an assumption is consistent with the Big Bang hypothesis (see EVIDENCE \#4). Such curves therefore should have originated suitably near the universe horizon created during Big Bang, which is characterized by the lowest initial proper-time. Thus, the root (12) identifies the proper-time of a (possibly virtual) graviton generated in coincidence, or immediately after, the Big Bang event. The remaining trajectories which are associated with a given observer identify instead (again possibly virtual) massive gravitons which are generated at later proper-times.

In order to be able to identify the proper-time $s$ also as a global observable it is necessary to require, in addition, that:

(4) For all observers which can be mutually connected by null geodetics (i.e., necessarily belong to the same light-cone) and for all semi-infinite geodesic curves which are associated with them, the corresponding initial proper-times $s_{0}$ are all positive or null.

(5) Among them for all observers there is again for each one possibly a non-unique "maximal length" geodetics with origin point $r^{\mu}\left(s_{0}\right)$ such that the condition (12) holds.

\subsection{Interpretation/Meaning of Proper-Time}

An important issue about proper-time concerns its possible interpretation and meaning. This concerns the customary interpretation occurring in the context of General Relativity [94,95], i.e., in terms of the Riemann distance on the space-time (geometric interpretation). Such an interpretation is based on Equations (9) and (10). However, it does not provide, by itself, a unique prescription for $s$. In fact, once the reference 4-position $r=r(s)$ (see Equation (11)) is prescribed, the precise value of $s$ depends both on the choice of the space-time curve on which it is measured and that of the reference 4-position $r_{1}=r\left(s_{1}\right)$ on the same curve. As shown above, these indeterminacies can be resolved if, for all observers belonging to the same light-cone, proper-time is the arc length measured along an arbitrary observer geodetics with origin point $r\left(s_{0}\right)$ and in particular along an observer's maximal geodetics having origin point $r\left(s_{o}=0\right)$ (CCG-theory geometric interpretation). Under the assumption of existence of massive gravitons, proper-time acquires also the further interpretation according to which, for all observers belonging to the same light-cone, it is the arc length of the worldline of a graviton measured between its origin point $r\left(s_{o}=0\right)$ and the observer position $r(s)=r$ (dynamic interpretation). Finally, proper-time can also be interpreted as a global classical observable realized by a 4-scalar, which can be measured by an arbitrary observer via an ideal measurement experiment or gedanken experiment (experimental interpretation).

Some additional remarks are in order concerning the role of proper-time in CQG-theory. First of all it must be stressed that the adoption of the proper-time parametrization permits recovering the customary concepts and formalism of standard quantum mechanics and relativistic quantum mechanics also for CQG-theory, which are associated with the Hamiltonian and Hamilton-Jacobi structures of the theory and the physical meaning of the quantum wave function. From one side the proper-time is consistent with the manifest covariance principle, since it is a 4-scalar (contrary to the coordinate time), while from the other side it indeed plays the role of "time" dynamical variable in terms of which dynamical evolution of quantum systems is parametrized, and therefore it represents also a convenient choice for the quantum theory itself. It follows that CQG-theory is truly founded on the notion of proper-time, which becomes necessary for the representation of the fundamental equations of CQG-theory and its physical interpretation. The role of the invariant proper-time is 
restored in CQG-theory as dynamical parameter, in difference with the marginal role played by coordinate time in loop quantum gravity (for a discussion of the issue see Ref. [95]).

\section{Covariant Classical/Quantum Gravity in the Extended Setting}

Let us now pose the problem of formulating the theory of covariant gravity in the extended setting of the type (3), i.e., in the general case of a non-stationary background metric tensor. This point, in view of the goals set in the paper, is crucial. The expectation in fact is that second-quantization effects arising due to non-linear quantum corrections of the background metric tensor might give rise to a possible explicit proper-time dependence of the same tensor field. For this purpose the prerequisite is to ascertain whether respectively the classical and quantum Hamiltonian structures determined in Refs. [3,4], which are set at the basis of CCG and CQG theories, can actually be preserved in such a case. This requires, more precisely, to uncover whether and under which conditions the validity of the principle of manifest covariance can be warranted. In the following subsections the issues are discussed in detail.

\subsection{The Classical Hamiltonian Structure of GR}

Let us first consider the extension of the reduced continuum Hamiltonian theory for GR and of the related classical Hamiltonian structure of GR developed in Ref. [3]. This is represented by a set $\left\{x_{R}, H_{R}\right\}$, formed by an appropriate 4-tensor canonical state $x_{R} \equiv\left(g_{\mu v}, \pi^{\mu v}\right)$ and an appropriate 4-scalar classical Hamiltonian density $H_{R}$. According to the same reference this is identified with the function

$$
H_{R} \equiv T_{R}+V,
$$

where the effective kinetic and the normalized effective potential density $T_{R}$ and $V$ are reported for completeness in Appendix A. Then, adopting for definiteness the Eulerian representation given in Ref. [6] and introducing the covariant s-derivative operator $\frac{d}{d s}$ (see Equation (18) below), by assumption the same Hamiltonian structure should generate the 4-tensor (continuum) GR-Hamilton equations:

$$
\left\{\begin{array}{c}
\frac{d g_{\mu v}}{d s}=\frac{\partial H_{R}}{\partial \pi^{\mu v}}, \\
\frac{d \pi^{\mu v}}{d s}=-\frac{\partial H_{R}}{\partial g_{\mu v}} .
\end{array}\right.
$$

In terms of Equations (A1) and (A3) (see Appendix A) these reduce to

$$
\left\{\begin{array}{c}
\frac{d g_{\mu v}}{d s}=\frac{\pi_{\mu v}}{\alpha L}, \\
\frac{d \pi^{\mu v}}{d s}=-\frac{\partial V}{\partial g_{\mu v}} .
\end{array}\right.
$$

Omitting possible implicit dependences (i.e., with respect to the tangent 4-vector $t \equiv\left\{t^{\alpha}\right\}$, see e.g., Equation (19) below and Ref. [4]) $H_{R}$ is assumed to be of the form $H_{R}=H_{R}\left(x_{R}, \widehat{g}, r, s\right)$, where $\widehat{g}$ is according to Equation (3). Then, by introducing a proper-time parametrization of the canonical state of the form

$$
x_{R} \equiv x_{R}(s) \equiv x_{R}(r(s), s),
$$

the same state is assumed to be subject to an initial condition of the type

$$
\left\{\begin{array}{c}
g_{\mu v}\left(s_{1}\right) \equiv g_{\mu v}^{(o)}\left(r\left(s_{1}\right), s_{1}\right), \\
\pi^{\mu v}\left(s_{1}\right) \equiv \pi^{(o) \mu v}\left(r\left(s_{1}\right), s_{1}\right),
\end{array}\right.
$$

being $s_{1} \geq s_{0}$ and $r\left(s_{1}\right)$ respectively an initial proper-time and a geodesic curve evaluated at the same proper-time. The mandatory requisites in order to preserve the Hamiltonian structure indicated above, i.e., for the validity of the canonical Equation (14), are that they should, at the same time: (a) be 
manifestly-covariant; (b) by prescription of the initial conditions (17), reduce identically for all $s \geq s_{0}$ to the non-vacuum Einstein field Equation (5); (c) that a corresponding classical Hamilton-Jacobi equation, equivalent to the canonical Equation (14), should hold.

As shown in Appendix B, the first requisite demands that the differential operator $\frac{d}{d s}$ in Equations (14) and (15), when written in Eulerian form in analogy to Ref. [6], should take the form

$$
\frac{d}{d s}=\left.\frac{d}{d s}\right|_{s}+\left.\frac{d}{d s}\right|_{r}
$$

Here the notation is as follows. First, $\left.\frac{d}{d s}\right|_{s} \equiv t^{\alpha} \nabla_{\alpha}$ identifies the directional covariant derivative, with

$$
t^{\alpha}=\left.\frac{d r^{\alpha}(s)}{d s} \equiv \frac{d}{d s}\right|_{s} r^{\alpha}(s)
$$

being the tangent to the geodetic curve $r(s) \equiv\left\{r^{\alpha}(s)\right\}$. Second, $\left.\frac{d}{d s}\right|_{r}$ denotes now the covariant s-partial derivative. When it operates on a 4 -scalar this coincides with the ordinary partial derivative, so that

$$
\left.\frac{d}{d s}\right|_{r}=\frac{\partial}{\partial s}
$$

and consequently in this case

$$
\frac{d}{d s}=\left.\frac{d}{d s}\right|_{s}+\frac{\partial}{\partial s} \equiv D_{s}
$$

with $D_{s}$ to be referred to as convective derivative. However, when acting on a second-order tensor it must be prescribed according to Equation (A8) (or equivalently Equation (A9)) reported in the Appendix B respectively for the countervariant and covariant components of a generic second-order 4-tensor. As a consequence one obtains respectively that the operator $\frac{d}{d s}$ acts so that

$$
\left\{\begin{array}{l}
\frac{d}{d s} x^{\alpha \beta}=D_{s} x^{\alpha \beta}-\frac{1}{2} x^{p q} \widehat{g}_{\mu p} \widehat{g}_{v q} \frac{\partial}{\partial s}\left(\widehat{g}^{\alpha \mu} \widehat{g}^{\beta v}\right), \\
\frac{d}{d s} x_{\alpha \beta}=D_{s} x_{\alpha \beta}-\frac{1}{2} x_{p q} \widehat{g}^{\mu p} \widehat{g}^{v q} \frac{\partial}{\partial s}\left(\widehat{g}_{\alpha \mu} \widehat{g}_{\beta v}\right) .
\end{array}\right.
$$

Thus, in particular, when $x^{\alpha \beta} \equiv \widehat{g}^{\alpha \beta}(r, s)$ or $x_{\alpha \beta} \equiv \widehat{g}_{\alpha \beta}(r, s)$, namely the covariant and countervariant components of background metric tensor are considered, it follows that the identities

$$
\begin{aligned}
& \frac{d}{d s} \widehat{g}_{\mu v}(r, s) \equiv 0 \\
& \frac{d}{d s} \widehat{g}^{\mu v}(r, s) \equiv 0
\end{aligned}
$$

necessarily hold, where $r \equiv r(s)$ denotes the (arbitrary) geodetics indicated above. Regarding the second requisite, once Equation (18) is set, then the same formally follows in a straightforward way. In fact, introducing the initial conditions

$$
\left\{\begin{array}{c}
g_{\mu v}\left(s_{o}\right) \equiv \widehat{g}_{\mu v}\left(r\left(s_{o}\right), s_{o}\right), \\
\pi^{\mu v}\left(s_{o}\right) \equiv \widehat{\pi}^{\mu v}\left(r\left(s_{o}\right), s_{o}\right)=0,
\end{array}\right.
$$

and by requiring that the corresponding extremal fields are such that $\widehat{x}_{R}(s) \equiv\left(\widehat{g}_{\mu v}(s), \widehat{\pi}^{\mu v}(s) \equiv 0\right)$, implies that thanks to the identities (23) and (24), Equation (14) becomes identically

$$
\left\{\begin{array}{c}
\frac{d \widehat{g}_{\mu v}}{d s} \equiv 0, \\
-\left.\frac{\partial V}{\partial g_{\mu v}}\right|_{g_{\mu v}(s)=\widehat{g}_{\mu v}(s)}=0 .
\end{array}\right.
$$


Hence, the second equation coincides identically with the Einstein field Equation (5). The implication is therefore that the same equations hold also in validity of non-stationary sources and consequently in the case of a non-stationary background field tensor. These conclusions overcome the conditions earlier stated in Ref. [3] which instead referred to the case of a stationary background field tensor.

Finally, let us consider the requirement of validity of the Hamilton-Jacobi equation indicated above, originally first established in Ref. [3] for the case of stationary background field tensors (see THM. 1 in the same reference). The question arises whether also in the non-stationary case of Equation (3) the set of PDEs represented by the classical GR-Hamilton Equation (14) should be equivalent to a single PDE to be referred to as GR-Hamilton-Jacobi equation, namely of the type

$$
\frac{d \mathcal{S}(g, \widehat{g}, r, s)}{d s}+H_{R}(g, \pi, \widehat{g}, r, s)=0,
$$

which holds for a 4-scalar function of the form $\mathcal{S}(g, \widehat{g}, r, s)$ (Hamilton principal function), with $\widehat{g} \equiv \widehat{g}(s)$ to be understood everywhere in the following as a non-stationary tensor of the type (3). In addition, due to the arbitrariness in the definition of the same function $\mathcal{S}(g, \widehat{g}, r, s)$, the latter should be prescribed so that: (1) first it results identically

$$
\pi^{\mu v}=\frac{\partial \mathcal{S}(g, \widehat{g}, r, s)}{\partial g_{\mu v}}
$$

with $\pi^{\mu v}$ being the canonical momentum conjugate to $g_{\mu v}$; (2) second, denoting by $\left(G_{\mu v} \equiv g_{\mu v}\left(s_{1}\right), P^{\mu v} \equiv \pi^{\mu v}\left(s_{1}\right)\right)$ the initial state prescribed according to the initial condition (17), the classical phase-function $\mathcal{S}(g, \widehat{g}, r, s)$ should actually depend functionally on the initial canonical state function $P$, to be identified either with the initial coordinate $P \equiv\left\{g_{\mu v}\left(s_{1}\right)\right\}$, the conjugate momentum $P \equiv\left\{\pi^{\mu v}\left(s_{1}\right)\right\}$ or more generally a function of both of them, i.e., to be of the form

$$
\mathcal{S}(g, \widehat{g}, r, s) \equiv \mathcal{S}(g, \widehat{g}, r, s ; P) .
$$

In addition, in order that the classical Hamilton-Jacobi equation actually warrants validity of Equation (14) the same Hamilton principal function should satisfy identically also the corresponding constraint equations

$$
\left\{\begin{array}{l}
Q_{\mu v}=\frac{\partial \mathcal{S}(g, \widehat{g}, r, r, s ; P)}{\partial P^{\mu v}}, \\
\left|\frac{\partial \mathcal{S}^{2}(g, \widehat{g}, r, s ; P)}{\partial g_{\mu \nu} \partial P^{\mu v}}\right| \neq 0,
\end{array}\right.
$$

with $Q_{\mu v}$ being a constant phase function, i.e., such that $D_{s} Q_{\mu v} \equiv 0$, and $\left|\frac{\partial \mathcal{S}^{2}(g, \widehat{g}, r, s)}{\partial g_{\mu \nu} \partial P^{\mu v}}\right|$ being the determinant of the matrix $\left\{\frac{\partial \mathcal{S}^{2}(g, \widehat{g}, r, s ; P)}{\partial g_{\mu \nu} \partial P^{* v}}\right\}$. The latter, as usual in Hamilton-Jacobi theory, is therefore required to be non-singular. To prove the validity of the GR-Hamilton equations let us evaluate first the partial derivative of Equation (27) with respect to $g^{i k}$, keeping constant both $\frac{\partial \mathcal{S}(g, \widehat{g}, r, s ; P)}{\partial g^{i \xi}}$ and $\Pi \equiv\left\{\Pi^{\mu \nu}\right\}$. This gives

$$
\begin{aligned}
& \frac{\partial}{\partial g_{i k}} H_{R}\left(g^{\beta \gamma}, \frac{\partial \mathcal{S}(g, \widehat{g}, r, s ; P)}{\partial g^{\prime \zeta}}, \widehat{g}, r, s\right)+ \\
& \frac{\partial}{\partial g_{i k}} \frac{d}{d s} \mathcal{S}(g, \widehat{g}, r, s ; P)=0
\end{aligned}
$$


where the covariant $s$-derivative $\frac{d}{d s} \equiv D_{s}$ acting on the 4 -scalar $\mathcal{S}(g, \widehat{g}, r, s ; P)$ is performed keeping $g \equiv\left\{g^{\mu \nu}\right\}$ and $P \equiv\left\{P^{\mu \nu}\right\}$ constant and therefore is necessarily prescribed according to Equations (18) and (20). In addition, the identities

$$
\begin{aligned}
\frac{\partial}{\partial g_{\mu \nu}} D_{s} \mathcal{S}(g, \widehat{g}, r, s ; P) & =\frac{d}{d s} \frac{\partial}{\partial g_{\mu \nu}} \mathcal{S}(g, \widehat{g}, r, s ; P), \\
\frac{\partial}{\partial g^{\mu \nu}} D_{s} \mathcal{S}(g, \widehat{g}, r, s ; P) & =\frac{d}{d s} \frac{\partial}{\partial g^{\mu \nu}} \mathcal{S}(g, \widehat{g}, r, s ; P),
\end{aligned}
$$

hold respectively for the counter- and covariant components, where on the lhs $D_{S}$ is identified with the operator (21). On the other hand upon denoting $\frac{\partial}{\partial g_{\mu \nu}} \mathcal{S}(g, \widehat{g}, r, s ; P) \equiv \pi^{\mu v}$ and $\frac{\partial}{\partial g^{\mu v}} \mathcal{S}(g, \widehat{g}, r, s ; P) \equiv \pi_{\mu v}$ (and identifying respectively $\pi^{\mu v} \equiv x^{\mu v}$ and $\pi_{\mu v} \equiv x_{\mu \nu}$ in Equation (A8) of Appendix B), it is obvious that in order to preserve the correct covariance properties of the previous equations the operator $\frac{d}{d s}$ appearing on the rhs of Equations (32) and (33) now must coincide with the covariant $s$-derivative acting on the counter- and covariant components of a second-order 4-tensor respectively. Therefore upon identifying $\frac{\partial}{\partial g_{\mu \nu}} \mathcal{S}(g, \widehat{g}, r, s ; P) \equiv \pi^{\mu v}$ the second equation in the GR-Hamilton Equation (14) is found to be implied by the classical Hamilton-Jacobi equation. The construction of the corresponding PDE for $\frac{d g_{\mu v}}{d s}$ is straightforward and analogous to that given in Ref. [3], thus implying the equivalence between the GR-Hamilton equations and the GR-Hamilton-Jacobi equation. The consequence is therefore that the classical Hamiltonian structure $\left\{x_{R}, H_{R}\right\}$ remains preserved also in the case of a non-stationary background metric tensor (3).

\subsection{GR-Hamilton-Jacobi Quantization}

Based on the validity of the classical GR-Hamilton equations as well the corresponding classical GR-Hamilton-Jacobi equation, it is now formally straightforward to carry out the analogous extension for covariant quantum gravity. The conclusion follows at once adopting the quantization approach developed in Ref. [5], i.e., achieved by means of the so-called Hamilton-Jacobi g-quantization. In detail, this is realized through the mapping

$$
\begin{aligned}
& g_{\mu \nu} \rightarrow g_{\mu \nu}^{(q)} \equiv g_{\mu \nu}, \\
& \pi_{\mu v} \equiv \frac{\partial \mathcal{S}(g, \widehat{g}, r, s ; P)}{\partial g^{\mu \nu}} \rightarrow \pi_{\mu \nu}^{(q)} \equiv-i \hbar \frac{\partial}{\partial g^{\mu \nu}} \\
& p \equiv-\frac{\partial \mathcal{S}(g, \widehat{g}, r, s ; P)}{\partial s} \rightarrow p^{(q)} \equiv-i \hbar \frac{d}{d s} \\
& H_{R}\left(g, \frac{\partial \mathcal{S}(g, \widehat{g}, r, s ; P)}{\partial g}, \widehat{g}(s), r, s\right) \rightarrow H_{R}^{(q)}
\end{aligned}
$$

with $g_{\mu \nu}^{(q)}, \pi_{\mu \nu}^{(q)}, p^{(q)}$ and $H_{R}^{(q)}$ denoting the corresponding quantum fields/operators. Accordingly, $\pi_{\mu \nu}^{(q)}$, $p^{(q)}$ denote the quantum canonical momenta conjugate to $g_{\mu v}^{(q)} \equiv g_{\mu v}$ and $s$ respectively, while

$$
\begin{aligned}
H_{R}^{(q)} & \equiv T_{R}^{(q)}(\pi, \widehat{g})+V \\
T_{R}^{(q)}(\pi, \widehat{g}) & =\frac{1}{2 \alpha L}\left(-i \hbar \frac{\partial}{\partial g^{\mu \nu}}\right)\left(-i \hbar \frac{\partial}{\partial g^{\mu \nu}}\right),
\end{aligned}
$$

are the quantum Hamiltonian operator (with $V$ being the effective potential prescribed according to the second equation of Equation (A1) given in Appendix A) and the quantum effective kinetic energy operator. The mapping realized by Equations (34)-(37) implies the simultaneous validity of the two fundamental commutator relations

$$
\left[\pi^{(q) \alpha \beta}, g_{\mu v}\right]=-i \hbar \delta_{\mu}^{\alpha} \delta_{v}^{\beta}
$$




$$
\left[p^{(q)}, s\right]=-i \hbar
$$

together with

$$
\left[g^{\alpha \beta}, g_{\mu v}\right]=\left[\pi^{(q) \alpha \beta}, \pi_{\mu v}^{(q)}\right]=0 .
$$

Here we notice that since both $\pi^{(q) \alpha \beta}$ and $g_{\mu \nu}^{(q)}$ are symmetric, Equation (40) holds for arbitrary permutations of the indexes. As a consequence, based on the classical Hamilton-Jacobi Equation (27) also the mapping

$$
\frac{d \mathcal{S}}{d s}+H_{R}=0 \Rightarrow\left\{p^{(q)}+H_{R}^{(q)}\right\} \psi(s)=0
$$

necessarily applies. This warrants the validity of the quantum-wave equation

$$
i \hbar \frac{d}{d s} \psi(s)=H_{R}^{(q)} \psi(s),
$$

to be denoted as CQG-wave equation, with $\frac{d}{d s}$ denoting again the total covariant $s$-derivative in Eulerian form defined by Equation (21). According to the notations of Ref. [4], and omitting possible implicit contributions, here $\psi(g, s) \equiv \psi(g, \widehat{g}, r, s)$, with $r=r(s)$, denotes, in principle for arbitrary $s$ belonging to the time axis $I \equiv \mathbb{R}$, the 4-scalar quantum wave function associated with a graviton particle. Furthermore, $g=\left\{g_{\mu v}\right\}$ is the quantum generalized-coordinate field which spans the 10-dimensional real vector space $U_{g} \subseteq \mathbb{R}^{10}$ of the same wave-function, i.e., the set on which the associated quantum probability density function $\rho(g, s)=|\psi(g, s)|^{2}$ (quantum PDF) is prescribed.

One notices that, as shown in Ref. [4], the CQG-wave Equation (44) can be represented in terms of an equivalent set of quantum hydrodynamic equations [4,5]. This requires the adoption of the Madelung representation

$$
\psi(g, \widehat{g}, r, s)=\sqrt{\rho(g, \widehat{g}, r, s)} \exp \left\{\frac{i}{\hbar} \mathcal{S}^{(q)}(g, \widehat{g}, r, s)\right\},
$$

where the quantum fluid fields $\left\{\rho, S^{(q)}\right\} \equiv\left\{\rho(g, \widehat{g}, r, s), \mathcal{S}^{(q)}(g, \widehat{g}, r, s)\right\}$ identify respectively the 4-scalar quantum PDF and quantum phase-function. Elementary algebra then shows that based on Equation (44) the same quantum fluid fields must satisfy the set of GR-quantum hydrodynamic equations (CQG-QHE) realized respectively by continuity and quantum Hamilton-Jacobi equations. Written again in Eulerian form these are given by

$$
\begin{aligned}
\frac{d \rho}{d s}+\frac{\partial}{\partial g_{\mu \nu}}\left(\rho V_{\mu \nu}\right) & =0, \\
\frac{d \mathcal{S}^{(q)}}{d s}+H^{(q)} & =0 .
\end{aligned}
$$

Here in both equations, according to the notation (21), $\frac{d}{d s} \equiv D_{s}$ [4]. Thus, in analogy with the classical phase-function $\mathcal{S}(g, \widehat{g}, r, s)$ (see Equations (29) and (30)) the quantum phase-function $\mathcal{S}^{(q)}$ is taken of the form

$$
\mathcal{S}^{(q)}(g, \widehat{g}, r, s) \equiv \mathcal{S}^{(q)}(g, \widehat{g}, r, s ; P) .
$$

In particular this means that $\mathcal{S}^{(q)}$ should depend smoothly on: (1) the initial tensor field $P \equiv\left\{P^{\mu v}\right\}$ to be considered independent of $(g, \widehat{g}, r, s)$ and constant in the sense $D_{s} P \equiv 0$; (2) the variables $(g, \widehat{g}, r, s)$. In addition, by assumption $\mathcal{S}^{(q)}$ is required to satisfy the constraint and regularity conditions determined respectively by:

$$
\left\{\begin{array}{l}
Q_{\mu v}=\frac{\partial \mathcal{S}^{(q)}\left(g, \widehat{g}, r_{,} ; P\right)}{\partial P^{\mu \nu}}, \\
\left|\frac{\partial \mathcal{S}^{(q) 2}(g, \widehat{g}, r, s ; P)}{\partial g_{\mu \nu} \partial P^{\mu v}}\right| \neq 0 .
\end{array}\right.
$$


Here again $\left|\frac{\partial \mathcal{S}^{(q) 2}(g, \widehat{g}, r, s ; P)}{\partial g_{\mu v} \partial P^{\mu v}}\right|$ denotes the determinant of the matrix $\left\{\frac{\partial \mathcal{S}^{(q) 2}(g, \widehat{g}, r, s ; P)}{\partial g_{\mu \nu} \partial P^{\mu v}}\right\}$ while both $P^{\mu v}$ and $Q_{\mu v}$ are assumed to be constant phase functions, i.e., such that $D_{s} P^{\mu v} \equiv 0$ and $D_{s} Q_{\mu v} \equiv 0$.

Furthermore, $V_{\mu v} \equiv V_{\mu v}(g, s)$ and $H^{(q)} \equiv H^{(q)}(g, s)$ denote respectively the quantum 4-tensor velocity field identified with

$$
V_{\mu \nu}=\frac{1}{\alpha L} \frac{\partial \mathcal{S}^{(q)}}{\partial g^{\mu \nu}}
$$

and the effective quantum Hamiltonian density

$$
H^{(q)}=\frac{1}{2 \alpha L} \frac{\partial \mathcal{S}^{(q)}}{\partial g^{\mu \nu}} \frac{\partial \mathcal{S}^{(q)}}{\partial g_{\mu \nu}}+V_{Q M}+V
$$

with $V \equiv V(g, s)$ being the effective potential defined according to Equation (A1) and $V_{Q M} \equiv V_{Q M}(g, s)$ being the Bohm effective quantum potential [96-98] given by

$$
V_{Q M} \equiv \frac{\hbar^{2}}{8 \alpha L} \frac{\partial \ln \rho}{\partial g^{\mu \nu}} \frac{\partial \ln \rho}{\partial g_{\mu \nu}}-\frac{\hbar^{2}}{4 \alpha L} \frac{\partial^{2} \rho}{\rho \partial g_{\mu \nu} \partial g^{\mu \nu}}
$$

with $\rho \equiv \rho(g, \widehat{g}, r, s) \equiv|\psi(g, \widehat{g}, r, s)|^{2}$ being the 4-scalar quantum PDF. Equation (44) is therefore manifestly covariant also in such a case. As such it retains its form under the action of arbitrary local point transformations which preserve the differential manifold of space-time. As such the same equation is appropriate for the treatment of problems of quantum gravity and quantum cosmology also in such extended framework.

We conclude that the CQG-wave Equation (44) and the equivalent set of CQG-QHE are both manifestly covariant also in validity of a non-stationary background metric tensor of the type (3). Therefore they both hold also in such a case.

\section{Hamiltonian Representation of the CQG-Quantum Hydrodynamics Equations}

In this section we intend to uncover a novel feature of CQG-theory not previously pointed out.

This concerns the Hamiltonian structure, which in analogy to nonrelativistic quantum mechanics $[15,99]$, is associated with the quantum wave equation (i.e., the CQG-wave Equation (44)) and the corresponding quantum Hamilton-Jacobi Equation (47). More precisely, we intend to show that such an equation is actually equivalent to a corresponding set of manifestly-covariant quantum Hamilton equations, thus establishing "de facto" a Hamiltonian structure analogous to that holding for the classical GR-Hamilton equations. Such a structure not only lies at the basis of CQG-theory developed in Refs. [3-6] but-as shown in Section 4-remains also preserved under the extended setting considered in the present paper. Given the equivalence between the classical GR equations and the corresponding GR-Hamilton-Jacobi equation established in Section 4 (see also the analogous one first pointed out in Ref. [3] in the case of stationary background) such a result is not surprising. Nevertheless, it is worth stressing its unique and peculiar feature which distinguishes CQG-theory from other previous non-manifestly covariant quantum theories of gravity, like the Wheeler-DeWitt equation [100].

In particular, the goal of this section is to display the quantum Hamilton equations and corresponding Hamiltonian structure represented by a set $\left\{x, H^{(q)}\right\}$ which are associated respectively with an appropriate 4-tensor canonical state $x \equiv\left(g_{\mu v}, \Pi^{\mu v}\right)$ and the 4-scalar effective quantum Hamiltonian density $H^{(q)}$. As shown below, here the second-order canonical 4-tensor momentum $\Pi^{\mu v}$ will be identified with

$$
\Pi^{\mu v}=\frac{\partial \mathcal{S}^{(q)}(g, \widehat{g}, r, s ; P)}{\partial g_{\mu v}}
$$


$\mathcal{S}^{(q)}(g, \widehat{g}, r, s ; P)$ being the quantum phase function of CQG-theory and $H^{(q)}$ the effective quantum Hamiltonian density prescribed according to Equation (51). In the current notation the latter is written as

$$
H^{(q)}(g, \Pi, \widehat{g}(r, s), r, s)=\frac{1}{2 \alpha L} \Pi^{\mu v} \Pi_{\mu v}+V_{Q M}+V .
$$

Then the following proposition holds.

Theorem 1 (Quantum Hamilton equations). In validity of the CQG-wave Equation (44) and the corresponding quantum Hamilton-Jacobi Equation (47), upon denoting $H(g, \Pi, r, s) \equiv H^{(q)}(g, \Pi, \widehat{g}, r, s)$, the canonical state $x \equiv\left(g_{\mu v}, \Pi^{\mu v}\right)$ satisfies identically the set of manifestly-covariant equations

$$
\left\{\begin{aligned}
\frac{d}{d s} g^{\mu v} & =\frac{\partial}{\partial \Pi_{\mu v}} H(g, \Pi, r, s), \\
\frac{d}{d s} \Pi_{\mu v} & =-\frac{\partial}{\partial g^{\mu v}} H(g, \Pi, r, s),
\end{aligned}\right.
$$

subject to the initial conditions

$$
\left\{\begin{aligned}
g_{\mu v}\left(s_{1}\right) & \equiv g_{\mu v}^{(o)}\left(r\left(s_{1}\right), s_{1}\right), \\
\Pi^{\mu v}\left(s_{1}\right) & \equiv \Pi_{(o)}^{\mu v}\left(r\left(s_{1}\right), s_{1}\right) .
\end{aligned}\right.
$$

Equations (55) and (56) are referred to here as Quantum Hamilton equations.

Proof. To prove the thesis one notices preliminarily that in validity of Equations (53) and (54), Equations (55) and (56) are realized by means of the set of equations

$$
\begin{aligned}
\frac{d}{d s} g^{\mu v} & =\frac{\Pi^{\mu v}}{\alpha L}, \\
\frac{d}{d s} \Pi_{\mu v} & =-\frac{\partial\left(V_{Q M}+V\right)}{\partial g^{\mu v}},
\end{aligned}
$$

to be solved subject to initial conditions of the form

$$
\left\{\begin{array}{c}
g^{\mu v}\left(s_{o}\right)=g_{(o)}^{\mu v}\left(r\left(s_{o}\right), s_{o}\right), \\
\Pi_{\mu v}\left(s_{o}\right)=\Pi_{\mu v}^{(o)}\left(r\left(s_{o}\right), s_{o}\right) .
\end{array}\right.
$$

The proof of the canonical Equations (55) and (56) is actually analogous to that reached in Section 4. Thus, in particular, letting $H(g, \Pi, r, s) \equiv H^{(q)}(g, \Pi, \widehat{g}, r, s)$, the second equation is obtained in two steps: first, by partial differentiation of the quantum Hamilton-Jacobi Equation (47) with respect to $g^{\mu \nu}$, while letting again $\widehat{g}_{\mu v}(s)$ as constant, namely

$$
\begin{aligned}
& \frac{\partial}{\partial g^{\mu v}} H(g, \Pi, r, s)+ \\
& \frac{d}{d s}\left[\frac{\partial \mathcal{S}^{(q)}(g, \widehat{g}, r, r, s)}{\partial g^{\mu v}}\right]=0,
\end{aligned}
$$

and second noting that $\frac{\partial}{\partial s}\left[\frac{\partial \mathcal{S}^{(q)}(g, \widehat{g}, r, s ; P)}{\partial g^{g v}}\right] \equiv \frac{d}{d s} \Pi_{\mu v}$. In a similar way, by evaluating the partial derivative with respect to $\frac{\partial \mathcal{S}^{(q)}(g, \widehat{g}, r, s ; P)}{\partial g^{\mu v}} \equiv \Pi_{\mu v}$ and keeping $\widehat{g}_{\mu v}(s), g^{\mu v}$ and $r^{\mu}$ as constants, gives

$$
\begin{aligned}
& \frac{\partial}{\partial \Pi_{\mu v}} H(g, \Pi, r, s)+ \\
& \frac{\partial}{\partial \Pi_{\mu v}} \frac{\partial \mathcal{S}^{(q)}(g, \widehat{g}, r, s ; P)}{\partial s}=0 .
\end{aligned}
$$


Then, the proof of Equation (53) follows by invoking the identity

$$
\begin{gathered}
\left.\frac{\partial}{\partial \frac{\partial \mathcal{S}^{(q)}(g, s)}{\partial g^{\mu \nu}}} \frac{\partial \mathcal{S}^{(q)}(g, \widehat{g}, r, s ; P)}{\partial s}\right|_{g^{\mu \nu}}= \\
\frac{\partial}{\partial \frac{\partial \mathcal{S}^{(q)}(g, s)}{\partial g^{\mu \nu}}} \frac{\partial \mathcal{S}^{(q)}(g, \widehat{g}, r, s ; P)}{\partial s}- \\
-\frac{d}{d s} g^{\beta \gamma} \frac{\partial}{\partial \frac{\partial \mathcal{S}^{(q)}(g, s)}{\partial g^{\mu \nu}}} \frac{\partial \mathcal{S}^{(q)}(g, \widehat{g}, r, s ; P)}{\partial g^{\beta \gamma}},
\end{gathered}
$$

where $\frac{\partial}{\partial \frac{\partial \mathcal{S}^{(q)}(g, \hat{g}, r, s ; \beta)}{\partial g^{l v}}} \frac{\partial \mathcal{S}^{(q)}(g, \widehat{g}, r, s ; P)}{\partial g^{\beta \gamma}}=\delta_{\beta \gamma}^{\mu v}$ and $\delta_{\beta \gamma}^{\mu v} \equiv \delta_{\beta}^{\mu} \delta_{\gamma}^{\nu}$. Notice that, since the first term on the rhs of Equation (62) vanishes identically, here $\frac{\partial \mathcal{S}^{(q)}(g, \widehat{g}, r, s ; P)}{\partial s}$ must be considered as independent of $\Pi_{\mu v}$, because they represent different canonical momenta. Finally, one notices that the dependence of the quantum phase-function $\mathcal{S}^{(q)}$ in terms of $P \equiv\left\{P^{\mu \nu}\right\}$ remains still indeterminate. This means that it can still be prescribed in such a way to satisfy identically the constraint equation

$$
\frac{\partial \mathcal{S}^{(q)}(g, \widehat{g}, r, s ; P)}{\partial P^{\mu v}} \equiv Q_{(o) \mu v}\left(r\left(s_{o}\right)\right)
$$

with $Q_{(o) \mu v}\left(r\left(s_{o}\right)\right)$ being the (still arbitrary) initial constant phase function prescribed in analogy to Equations (59). As a consequence the 4-scalar quantum hydrodynamic Equation (47), in analogy with the GR-Hamilton-Jacobi equation discussed above in Section 4 (see Equation (27)), can indeed be interpreted in a proper sense as a Hamilton-Jacobi equation, i.e., as generating a corresponding set of Hamilton equations. Q.E.D.

\section{Quantum Modified Einstein Field Equations}

Concerning the quantum Hamilton Equation (55) the fundamental question to be answered is whether they actually admit a particular realization which is analogous to the classical Einstein Equation (5), for a suitable choice of the initial conditions (56) and in close analogy with the classical GR-Hamilton Equation (14) for which such a property was first pointed out in Ref. [3]. Being based on the quantum Hamilton Equation (55), the tensor components of such an equation will be referred to as quantum-modified Einstein field equations.

Given the formal analogy of the two sets of Hamiltonian equations, i.e., (14) and (55), both holding in validity of the extended functional setting (3), the following result holds.

Theorem 2 (Quantum-modified Einstein field equations). Let us assume validity of the initial-value problem represented by the quantum Hamilton Equation (55) and the initial conditions (56). For this purpose let us impose that the initial conditions are prescribed requiring

$$
\left\{\begin{array}{c}
g_{\mu v}\left(r\left(s_{o}\right), s_{o}\right)=\widehat{g}_{\mu v}\left(r\left(s_{o}\right), s_{o}\right), \\
\Pi^{\mu v}\left(r\left(s_{o}\right), s_{o}\right) \equiv 0,
\end{array}\right.
$$

("extremal" initial conditions) and that at the initial proper-time $s_{0}, \widehat{g}_{\mu v}(r(s), s)$ is solution of the PDE

$$
\left.\frac{\partial}{\partial g^{\mu \nu}}\left[V(g, \widehat{g}, r, s)+V_{Q M}(g, s)\right]\right|_{\substack{g=\widehat{g} \\ s=s_{o}}}=0
$$

(constraint equation). Then the following propositions hold: 
$\left(P 2_{1}\right)$ The quantum Hamilton equations in the general case of non-vacuum configuration reduce to the single extremal tensor equation

$$
\left.\frac{\partial}{\partial g^{\mu \nu}}\left[V(g, \widehat{g}, r, s)+V_{Q M}(g, s)\right]\right|_{g=\widehat{g}}=0 .
$$

$\left(\mathrm{P}_{2}\right)$ In the case of vacuum, namely letting

$$
V(g, \widehat{g}, r, s)=V_{o}(g, \widehat{g}, r, s),
$$

and setting $\Lambda=\Lambda_{\text {bare }}$ in Equation (A3), the extremal tensor Equation (66) becomes

$$
\widehat{R}_{\mu v}-\frac{1}{2}\left[\widehat{R}-2 \Lambda_{\text {bare }}\right] \widehat{g}_{\mu \nu}(r, s)=B_{\mu v}(r, s),
$$

which identifies the quantum-modified Einstein equation, where $B_{\mu v}$, prescribed in terms of the Bohm interaction potential $V_{Q M}$ as

$$
B_{\mu v}(r, s) \equiv-\left.\frac{1}{\alpha L} \frac{\partial}{\partial g} V_{Q M}(g, s)\right|_{g=\widehat{g}(r, s)},
$$

is referred to as Bohm source tensor field.

Proof. To reach the proof of $\mathrm{P} 2{ }_{1}$ we first obtain an equivalent explicit representation for the quantum Hamilton Equation (55) holding in case of validity of the extended representation (3). This follows thanks to Equation (A9) and the prescription of the covariant s-derivative given by Equation (18). Thus one finds that the same equations become:

$$
\left\{\begin{array}{c}
D_{s} g^{\mu v}-g_{\alpha}^{\mu} \frac{\partial}{\partial s}\left(\widehat{g}^{v \alpha}\right)=\frac{\Pi^{\mu v}}{\alpha L}, \\
D_{s} \Pi_{\mu v}-\Pi_{\mu}^{\alpha} \frac{\partial}{\partial s}\left(\widehat{g}_{v \alpha}\right) \stackrel{=}{=} \\
-\frac{\partial}{\partial g^{\mu \nu}}\left[V(g, \widehat{g}, r, s)+V_{Q M}(g, s)\right],
\end{array}\right.
$$

where

$$
\left\{\begin{aligned}
g_{\alpha}^{\mu} \frac{\partial}{\partial s}\left(\widehat{g}^{v \alpha}\right) & =g_{\alpha}^{\mu} D_{s}\left(\widehat{g}^{v \alpha}\right), \\
\prod_{\mu}^{\alpha} \frac{\partial}{\partial s}\left(\widehat{g}_{v \alpha}\right) & =\Pi_{\mu}^{\alpha} D_{s}\left(\widehat{g}_{v \alpha}\right) .
\end{aligned}\right.
$$

Hence, straightforward algebra delivers

$$
\left\{\begin{array}{c}
\widehat{g}^{\mu \alpha} D_{s} g_{\alpha}^{v}=\frac{\Pi^{\mu v}}{\alpha L}, \\
\widehat{g}_{v \alpha} D_{s} \Pi_{\mu}^{\alpha}= \\
-\frac{\partial}{\partial g^{\mu v}}\left[V(g, \widehat{g}, r, s)+V_{Q M}(g, s)\right],
\end{array}\right.
$$

implying, in turn, that the following equivalent explicit representation must hold for the same equations

$$
\left\{\begin{array}{c}
D_{s} g_{\alpha}^{v}=\frac{\Pi_{\alpha}^{v}}{\alpha L}, \\
D_{s} \Pi_{v}^{\alpha}=-\frac{\partial}{\partial g_{\alpha}^{v}}\left[V(g, \widehat{g}, r, s)+V_{Q M}(g, s)\right] .
\end{array}\right.
$$

As a second step one notices that the requirement that

$$
\forall s \in I: g_{\alpha}^{v}(r(s), s)=\widehat{g}_{\alpha}^{v}(r(s), s)
$$

is obviously equivalent to require that Equation (66) must hold identically. The implication, however, is that to reach the thesis it is actually necessary to prove that the initial conditions (64) are actually 
equivalent to the validity of proposition (74). That this is indeed the case follows in fact by direct inspection of Equation (73), since the constraint Equation (65) implies that

$$
\left.D_{s} \Pi_{v}^{\alpha}\right|_{s=s_{0}}=0,
$$

while the initial conditions (64) warrant that $\left.\prod_{v}^{\alpha}\right|_{s=s_{0}}=0$ too. Hence, for all $s \geq s_{o}$ it is identically vanishing as $D_{s} g_{\alpha}^{v}$, which means that the proposition (74) is necessarily true. As a consequence the validity of the extremal tensor Equation (66) remains warranted too. The proof of $\mathrm{P} 22$ then follows by elementary algebra once the condition (67) is imposed in Equation (66), which yields as well the representation of the Bohm source tensor field $Q_{\mu \nu}(s)$. Q.E.D.

The main implication arising from THM. 2 is the appearance of a quantum contribution to the stress energy tensor, denoted here $\widehat{T}_{\mu \nu}^{(q)}$. In the context of CQG-theory this is prescribed in terms of the Bohm source tensor field $B_{\mu v}$, so that

$$
B_{\mu v} \equiv \kappa \widehat{T}_{\mu v}^{(q)}
$$

with $\kappa$ being again the universal constant (6). The tensor field $B_{\mu v}$ is therefore ascribed to the effect of Bohm vacuum self-interaction acting on gravitons, and more precisely, as shown below (see Equation (146)), to corresponding quantum vacuum energy fluctuations produced by gravitons. The contribution associated with $B_{\mu \nu}$ defined by Equation (76) in the quantum-modified Einstein field Equation (68) realizes a space-time second-quantization effect, namely a quantum correction to the classical metric tensor arising from a non-linear quantum interaction of the gravitational field with itself. According to this picture, the background metric tensor solution of Equation (68) is affected by quantum corrections, and this distinguishes the second-quantization framework from the first-quantization one, where instead the background metric tensor is prescribed to be a purely-classical space-time field tensor.

One notices that Equation (68) coincides functionally with the set (5) of classical Einstein equations holding for the components of the background field tensor. Hence, Equation (68) will be referred to as quantum-modified Einstein field equations. Indeed, the two equations coincide once the tensor field $T_{\mu \nu}$ is replaced with the quantum field $B_{\mu v}(s)$. The notable difference arising in Equation (68) lies in the non-stationary character of the quantum source term, i.e., its explicit dependence on proper-time. Therefore in the present context all the tensor fields, including the background metric tensor $\widehat{g} \equiv\left\{\widehat{g}_{\mu v}\right\}$, the Ricci tensor $\widehat{R}_{\mu \nu}$ and the Ricci 4-scalar $\widehat{R} \equiv \widehat{g}^{\alpha \beta}(r, s) \widehat{R}_{\alpha \beta}$ are necessarily to be considered as non-stationary too.

Nevertheless, an additional difficulty arises due to the intrinsic quantum origin of the Bohm source tensor field $Q_{\mu v}$, for which the prescription of the quantum probability density $\rho \equiv \rho(g, \widehat{g}, r, s)$ is needed. Its determination requires in fact the explicit solution of the CQG-wave Equation (44) in a highly non-linear second quantization picture (i.e., in which also the background field tensor must be consistently evaluated). In fact, the crucial issue is that the latter must be consistently determined by means of the quantum-modified Einstein field equations themselves. To unfold these tasks and achieve the construction of explicit solutions of the CQG-wave equation the Generalized Lagrangian Path Approach (GLP-approach) recently developed in Ref. [6] will be adopted. For this purpose, as a starting point, the generalization of the GLP-approach to the extended functional setting adopted here is performed.

\section{GLP-Approach in the Extended Functional Setting}

In this section we show that also the GLP-approach developed in Ref. [6] remains valid for the extended functional setting (3) and actually fulfills the property of manifest covariance also in such a case. Let us start recalling, for this purpose, that the GLP-approach crucially depends on the notion of 
Generalized Lagrangian Path (GLP), i.e., the integral curve $\left\{\delta G_{L}(s) \equiv \delta G_{L \mu v}(r(s), s), \forall s \in I\right\}$ which is determined by the GLP-initial-value problem

$$
\left\{\begin{array}{c}
\frac{d}{d s} \delta G_{L \mu v}(s)=V_{\mu v}\left(G_{L}(s), \Delta g, r(s), s\right), \\
\delta G_{L \mu v}\left(s_{1}\right)=\delta g_{L \mu v}\left(s_{1}\right)-\Delta g_{\mu v}\left(s_{1}\right) \\
\delta g_{L \mu \nu}\left(s_{1}\right) \equiv \delta g_{L \mu v}^{(o)}
\end{array}\right.
$$

More precisely, here $\delta g_{L \mu \nu}(s)$ and $\delta G_{L \mu v}(s)$ identify respectively, according to Ref. [6], the deterministic Lagrangian Path (LP) and stochastic Generalized Lagrangian Path (GLP). We remark that in the extended functional setting $\frac{d}{d s}$ identifies the covariant $s$-derivative (18) while $r(s)$ denotes again, also in such a context, an arbitrary geodesic trajectory with $s_{1} \geq s_{0}$ being in principle an arbitrary initial proper-time along it. In particular in the following $r(s)$ and $s_{1}$ can always be identified respectively with a maximal observer's geodetics and, upon requiring

$$
s_{1}=s_{0}=0,
$$

with the Big Bang proper-time (12). Furthermore, according to the notations of Ref. [6] and consistent with Equation (50), $\delta g_{\mu \nu}^{(o)}$ and $V_{\mu \nu}\left(G_{L}(s), \Delta g, r(s), s\right)$ denote respectively a deterministic initial tensor field and the quantum tensor velocity field

$$
V^{\mu v}\left(G_{L}(s), \Delta g, r(s), s\right)=\frac{1}{\alpha L} \frac{\partial \mathcal{S}^{(q)}\left(G_{L}(s), \Delta g, r(s), s ; P\right)}{\partial \delta g_{L \mu \nu}},
$$

where $\Delta g \equiv\left\{\Delta g_{\mu \nu}\right\}$ identifies the stochastic displacement 4-tensor

$$
\Delta g_{\mu \nu}(s) \equiv \delta g_{L \mu \nu}(s)-\delta G_{L \mu \nu}(s) .
$$

\subsection{Formal Solution of GLP in the Extended Functional Setting}

It is obvious "a priori" that the solution of Equations (77) must depend on the functional setting of the background field tensor, i.e., again on the validity of the requirement (3). Nevertheless a formal exact solution of the same equations can directly be recovered also in such a case. In fact, upon denoting respectively

$$
\left\{\begin{array}{c}
\delta g_{L v}^{\alpha}(s)=\widehat{g}^{\mu \alpha}(r(s), s) \delta g_{L \mu v}(r(s), s), \\
\delta G_{L v}^{\alpha}(s)=\widehat{g}^{\mu \alpha}(r(s), s) \delta G_{L \mu v}(r(s), s), \\
\Delta g_{v}^{\alpha}(s)=\widehat{g}^{\mu \alpha}(r(s), s) \Delta g_{\mu v}(s), \\
V_{v}^{\alpha}\left(G_{L}(s), \Delta g, s\right)=\widehat{g}^{\mu \alpha}(r, s) V_{\mu v}\left(G_{L}(s), \Delta g, s\right),
\end{array}\right.
$$

one can show that the following result applies.

Theorem 3 (Construction of a formal representation of GLP). Regarding the GLP-initial-value problem (77) the following propositions apply:

$\left(P 3_{1}\right)$ Upon integration, Equation (77) actually delivers formal exact solutions both for $\delta g_{L v}^{\alpha}(s)$ and $\delta G_{L v}^{\alpha}(s)$. These are realized respectively by the initial-value problems

$$
\left\{\begin{array}{c}
\delta g_{L v}^{\alpha}(s)=\delta g_{L v}^{\alpha}\left(s_{o}\right)+\int_{s_{1}}^{s} d s^{\prime} V_{v}^{\alpha}\left(G_{L}\left(s^{\prime}\right), \Delta g, r\left(s^{\prime}\right), s^{\prime}\right), \\
\delta g_{L v}^{\alpha}\left(s_{o}\right) \equiv \delta g_{L v}^{\alpha(o)},
\end{array}\right.
$$


and

$$
\left\{\begin{array}{c}
\delta G_{L v}^{\alpha}(s)=\delta G_{L v}^{\alpha}\left(s_{o}\right)+\int_{s_{1}}^{s} d s^{\prime} V_{v}^{\alpha}\left(G_{L}\left(s^{\prime}\right), \Delta g, r\left(s^{\prime}\right), s^{\prime}\right) \\
\delta G_{L v}^{\alpha}\left(s_{o}\right)=\delta g_{L v}^{\alpha}\left(s_{0}\right)-\Delta g_{v}^{\alpha}\left(s_{o}\right), \\
\delta g_{L v}^{\alpha}\left(s_{o}\right) \equiv \delta g_{L v}^{\alpha(o)}
\end{array}\right.
$$

where

$$
V_{v}^{\alpha}\left(G_{L}(s), \Delta g, r(s), s\right)=\frac{1}{\alpha L} \frac{\partial \mathcal{S}^{(q)}\left(G_{L}(s), \Delta g, r(s), s ; P\right)}{\partial \delta g_{L \alpha}^{v}(s)} .
$$

These equations imply that by construction the stochastic displacement 4-tensor $\Delta g_{v}^{\mu}(s)$ must be a constant, i.e., such that for all $s, s_{o} \in I$

$$
\Delta g_{v}^{\mu}(s)=\Delta g_{v}^{\mu}\left(s_{o}\right) \equiv \Delta g_{v}^{\mu},
$$

while $\delta g_{L v}^{\alpha}(s)$ and $\delta G_{L v}^{\alpha}(s)$ are related by means of the transformation

$$
\delta G_{L v}^{\alpha}(s)=\delta g_{L v}^{\alpha}(s)-\Delta g_{v}^{\alpha} .
$$

$\left(P 3_{2}\right)$ Equations (82) and (83) can once again be represented in terms of the covariant components $\delta G_{L \mu v}(s)$ (and similarly $\delta g_{L \mu \nu}(s)$ ) yielding

$$
\begin{aligned}
& \delta G_{L \mu \nu}(s)=\widehat{g}_{\mu \alpha}(r(s), s) \delta G_{L v}^{\alpha}\left(s_{o}\right)+ \\
& +\widehat{g}_{\mu \alpha}(r(s), s) \int_{s_{1}}^{s} d s^{\prime} V_{v}^{\alpha}\left(G_{L}\left(s^{\prime}\right), \Delta g, r\left(s^{\prime}\right), s^{\prime}\right) .
\end{aligned}
$$

$\left(\mathrm{P}_{3}\right)$ Finally, in validity of Equation (2) (stationary background metric tensor), the same equations recover identically the form determined previously in Ref. [6], namely

$$
\begin{aligned}
& \delta g_{L \mu \nu}(s)=\delta g_{L \mu \nu}\left(s_{0}\right)+ \\
& +\int_{s_{1}}^{s} d s^{\prime} V_{\mu \nu}\left(G_{L}\left(s^{\prime}\right), \Delta g, r\left(s^{\prime}\right), s^{\prime}\right),
\end{aligned}
$$

with $\Delta g_{\mu v}(s)$ being such that identically

$$
\Delta g_{\mu \nu}(s)=\Delta g_{\mu v}\left(s_{0}\right) \equiv \Delta g_{\mu \nu},
$$

and

$$
\delta G_{L \mu v}(s)=\delta g_{L \mu v}(s)-\Delta g_{\mu \nu} .
$$

Proof. The proof of the previous statements follows by straightforward algebra. For this purpose one notices first that Equation (77) can be equivalently written as

$$
\begin{aligned}
& D_{s} \delta G_{L \mu \nu}(s)=V_{\mu \nu}\left(G_{L}(s), \Delta g, s\right)+ \\
& \frac{1}{2} \delta G_{L p q}(s) \widehat{g}^{\mu^{\prime} p}(r, s) \widehat{g}^{\nu^{\prime} q}(r, s) \frac{\partial}{\partial s}\left(\widehat{g}_{\mu \mu^{\prime}}(r, s) \widehat{g}_{v v^{\prime}}(r, s)\right),
\end{aligned}
$$

where it is obvious also that $\frac{\partial}{\partial s}\left(\widehat{g}_{\mu \mu^{\prime}}(r, s) \widehat{g}_{v v^{\prime}}(r, s)\right) \equiv D_{s}\left(\widehat{g}_{\mu \mu^{\prime}}(r, s) \widehat{g}_{v v^{\prime}}(r, s)\right)$ and furthermore

$$
\begin{aligned}
& \frac{1}{2} \delta G_{L p q}(s) \widehat{g}^{\mu^{\prime} p}(r, s) \widehat{g}^{\nu^{\prime} q}(r, s) \frac{\partial}{\partial s}\left(\widehat{g}_{\mu \mu^{\prime}}(r, s) \widehat{g}_{v v^{\prime}}(r, s)\right) \\
& =\delta G_{L v}^{\mu^{\prime}}(s) D_{s}\left(\widehat{g}_{\mu \mu^{\prime}}(r, s)\right) .
\end{aligned}
$$


Denoting $\delta G_{L v}^{\mu^{\prime}}(s) \widehat{g}_{\mu \mu^{\prime}}(r, s)=\delta G_{L \mu \nu}(s)$, the Leibnitz differentiation rule requires the identity

$$
\begin{aligned}
& \delta G_{L v}^{\mu^{\prime}}(s) D_{s}\left(\widehat{g}_{\mu \mu^{\prime}}(r, s)\right)=D_{s}\left(\delta G_{L v}^{\mu^{\prime}}(s) \widehat{g}_{\mu \mu^{\prime}}(r, s)\right) \\
& -\widehat{g}_{\mu \mu^{\prime}}(r, s) D_{s}\left(\delta G_{L v}^{\mu^{\prime}}(s)\right)
\end{aligned}
$$

to hold. Hence, Equation (91) finally yields

$$
\begin{aligned}
& D_{s} \delta G_{L \mu \nu}(s)=V_{\mu v}\left(G_{L}(s), \Delta g, s\right)+ \\
& D_{s} \delta G_{L \mu \nu}(s)-\widehat{g}_{\mu \mu^{\prime}}(r, s) D_{s}\left(\delta G_{L v}^{\mu^{\prime}}(s)\right),
\end{aligned}
$$

which also in turn implies

$$
V_{\mu \nu}\left(G_{L}(s), \Delta g, s\right)-\widehat{g}_{\mu \mu^{\prime}}(r, s) D_{s}\left(\delta G_{L v}^{\mu^{\prime}}(s)\right)=0 .
$$

Therefore, in the previous equation, upon multiplying tensorially term by term by $\hat{g}^{\mu \alpha}(r, s)$ and noting that $\widehat{g}^{\mu^{\prime} \alpha}(r, s) \widehat{g}_{\mu \mu^{\prime}}(r, s)=\delta_{\mu}^{\alpha}$ and $\widehat{g}^{\mu \alpha}(r, s) V_{\mu v}\left(G_{L}(s), \Delta g, s\right)=V_{v}^{\alpha}\left(G_{L}(s), \Delta g, s\right)$, the differential Equation (95) implies

$$
D_{s}\left(\delta G_{L v}^{\alpha}(s)\right)=V_{v}^{\alpha}\left(G_{L}(s), \Delta g, s\right),
$$

which, in turn, upon integration delivers the integral Equation (83) too (or equivalently Equation (87)). Finally, the proof of $\mathrm{P}_{3}$ follows by noting that in case of a stationary background metric tensor Equation (91) reduces to

$$
D_{s} \delta G_{L \mu \nu}(s)=V_{\mu v}\left(G_{L}(s), \Delta g, s\right),
$$

thus implying in turn Equation (88). Q.E.D.

One notices, however, that the GLP initial-value problem (77) can be equivalently replaced with

$$
\left\{\begin{array}{c}
\frac{d}{d s} \delta G_{L \mu v}(s)=V_{\mu v}\left(G_{L}(s), \Delta g, s\right) \\
\delta G_{L \mu v}(s)=\delta g_{L \mu v}(s)-\Delta g_{\mu \nu} \\
\delta g_{L \mu v}(s)=\delta g_{L \mu \nu}
\end{array}\right.
$$

with $\delta g_{L \mu \nu}$ prescribing now the initial condition (associated with the deterministic Lagrangian Path). Equation (98) admits the formal solution

$$
\left\{\begin{array}{c}
\delta G_{L v}^{\alpha}(s)=\delta G_{L v}^{\alpha}\left(s_{1}\right)+\int_{s_{1}}^{s} d s^{\prime} V_{v}^{\alpha}\left(G_{L}\left(s^{\prime}\right), \Delta g, r\left(s^{\prime}\right), s^{\prime}\right), \\
\delta G_{L v}^{\alpha}(s)=\delta g_{L v}^{\alpha}-\Delta g_{v}^{\alpha},
\end{array}\right.
$$

while correspondingly

$$
\left\{\begin{array}{c}
\delta g_{L v}^{\alpha}(s)=\delta g_{L v}^{\alpha}\left(s_{1}\right)+\int_{s_{1}}^{s} d s^{\prime} V_{v}^{\alpha}\left(G_{L}\left(s^{\prime}\right), \Delta g, r\left(s^{\prime}\right), s^{\prime}\right), \\
\delta g_{L v}^{\alpha}(s)=\delta g_{L v}^{\alpha} .
\end{array}\right.
$$

As a consequence the stochastic displacement 4-tensor defined by Equation (80) can also be equivalently represented as

$$
\Delta g_{v}^{\mu}(s) \equiv \delta g_{L v}^{\mu}-\delta G_{L v}^{\mu}(s),
$$


where $\delta g_{L v}^{\mu} \equiv g_{L v}^{\mu}-\widehat{g}_{v}^{\mu}(r, s)$ is considered prescribed and $\delta G_{L v}^{\mu}(s)$ is a function of the proper-time $s$. Then, introducing as in Ref. [6] the Lagrangian derivative realized by the operator

$$
\left.\frac{D}{D s} \equiv \frac{d}{d s}\right|_{\delta g_{L \mu \nu}(s)}+V_{v}^{\mu}\left(G_{L}(s), \Delta g, r, s\right) \frac{\partial}{\partial \delta g_{L v}^{\mu}},
$$

and upon denoting $\left.\frac{d}{d s}\right|_{\delta g_{L v}^{\mu}(s)} \equiv \frac{d}{d s}$ and invoking also Equation (98), one finds that the differential identity

$$
\begin{aligned}
\frac{D}{D s} \Delta g_{v}^{\mu}(s)= & V_{v}^{\mu}\left(G_{L}(s), \Delta g, r, s\right)- \\
& V_{v}^{\mu}\left(G_{L}(s), \Delta g, r, s\right) \equiv 0
\end{aligned}
$$

necessarily holds.

\subsection{Properties of Polynomial GLP-Solutions of the Hamilton-Jacobi Equation}

Next, in analogy to Ref. [6] let us consider polynomial GLP-solutions for the quantum phase-function $\mathcal{S}^{(q)}\left(G_{L}(s), \Delta g, r, s\right)$, namely represented in terms of a polynomial "harmonic" representation, i.e., determined by means of a second-degree polynomial of the form

$$
\begin{aligned}
& \mathcal{S}^{(q)}\left(G_{L}(s), \Delta g, r, s ; P\right) \equiv \\
& \frac{a_{p q}^{\alpha \beta}(s)}{2} \Delta g_{\alpha \beta} \Delta g^{p q}+b_{\alpha \beta}(s) \Delta g^{\alpha \beta}+c(s) .
\end{aligned}
$$

Here $a_{\mu v}^{\alpha \beta}(s), b_{\mu v}(s)$ and $c(s)$ denote respectively suitable real 4-tensors and a 4-scalar functions of $s$ to be determined in terms of the quantum H-J Equation (47) recalled above. In particular, consistent again with Ref. [6] and upon denoting $\delta_{p q}^{\alpha \beta} \equiv \delta_{p}^{\alpha} \delta_{q}^{\beta}$, the tensor coefficients $a_{p q}^{\alpha \beta}(s)$ are taken of the form

$$
a_{p q}^{\alpha \beta}(s)=\frac{1}{2}\left[a_{(o)}(s) \delta_{p q}^{\alpha \beta}+a_{(1)}(s) \widehat{g}_{p q}(s) \widehat{g}^{\alpha \beta}(s)\right],
$$

with $a_{(o)}(s)$ and $a_{(1)}(s)$ being appropriate 4-scalar functions. Since

$$
\left\{\begin{array}{c}
\delta_{p q}^{\alpha \beta} \Delta g_{\alpha \beta} \Delta g^{p q}=\Delta g_{\alpha \beta} \Delta g^{\alpha \beta}, \\
\widehat{g}_{p q}(s) \widehat{g}^{\alpha \beta}(s) \Delta g_{\alpha \beta} \Delta g^{p q}=\Delta g_{\alpha}^{\alpha} \Delta g_{\beta^{\prime}}^{\beta}
\end{array}\right.
$$

from Equation (104) it follows

$$
\alpha_{p q}^{\alpha \beta}(s) \Delta g_{\alpha \beta} \Delta g^{p q}=\frac{1}{2}\left[a_{(o)}(s) \Delta g_{\alpha \beta} \Delta g^{\alpha \beta}+a_{(1)}(s) \Delta g_{\alpha}^{\alpha} \Delta g_{\beta}^{\beta}\right],
$$

and therefore

$$
\begin{aligned}
& \mathcal{S}^{(q)}\left(G_{L}(s), \Delta g, s\right)= \\
& \frac{1}{4}\left[a_{(o)}(s) \Delta g_{\alpha \beta} \Delta g^{\alpha \beta}+a_{(1)}(s) \Delta g_{\alpha}^{\alpha} \Delta g_{\beta}^{\beta}\right]+ \\
& b_{\alpha \beta}(s) \Delta g^{\alpha \beta}+c(s) .
\end{aligned}
$$

Notice furthermore that here for consistency with Equations (49) and the invariance property of the displacement tensor field $\Delta g_{v}^{\mu}(s)$ :

$$
\Delta g_{v}^{\mu}(s)=\Delta g_{v}^{\mu}\left(s_{1}\right) \equiv \delta g_{L v}^{\mu}\left(s_{1}\right)-\delta G_{L v}^{\mu}\left(s_{1}\right),
$$


the constant tensor field $P_{v}^{\mu}$ can always be identified with

$$
P_{v}^{\mu}=\delta g_{L v}^{\mu}\left(s_{1}\right) .
$$

In particular, this warrants that Equations (49) can indeed by suitably fulfilled by a particular solution of the form (108). On the same grounds the effective quantum Hamiltonian density (51) can equivalently be represented as

$$
H^{(q)}=\frac{1}{2 \alpha L} \frac{\partial \mathcal{S}^{(q)}}{\partial \delta g_{L \nu}^{\mu}} \frac{\partial \mathcal{S}^{(q)}}{\partial \delta g_{L \mu}^{\nu}}+V_{Q M}+V,
$$

where

$$
\frac{\partial \mathcal{S}^{(q)}}{\partial \delta g_{v}^{\mu}}=p(s)\left[a_{(o)}(s) \Delta g_{\mu}^{v}+a_{(1)}(s) \delta_{\mu}^{v} \Delta g_{\beta}^{\beta}\right]+p(s) b_{\mu}^{v}(s),
$$

and

$$
\begin{aligned}
& {\left[a_{(o)}(s) \Delta g_{\mu}^{v}+a_{(1)}(s) \delta_{\mu}^{v} \Delta g_{\beta}^{\beta}\right]\left[a_{(o)}(s) \Delta g_{v}^{\mu}+\right.} \\
& \left.a_{(1)}(s) \delta_{v}^{\mu} \Delta g_{\beta}^{\beta}\right]=a_{(o)}^{2}(s) \Delta g_{\mu}^{v} \Delta g_{v}^{\mu}+ \\
& {\left[4 a_{(1)}^{2}(s)+2 a_{(o)}(s) a_{(1)}(s)\right] \Delta g_{\alpha}^{\alpha} \Delta g_{\beta}^{\beta} .}
\end{aligned}
$$

Then one can show that the following result applies.

Theorem 4 (Polynomial GLP-solutions of the Hamilton-Jacobi equation in the case of vacuum). Regarding the existence of polynomial GLP-solutions of the quantum Hamilton-Jacobi Equation (47) in the case of vacuum which hold in validity of the extended functional setting (3) the following propositions apply:

$\left(\mathrm{P}_{1}\right)$ The quantum-modified Einstein field Equation (68) are recovered by requiring the identical validity of the extremal equation

$$
\left.\frac{\partial}{\partial \Delta g^{\mu \nu}}\left[V_{o}(g+\Delta g)+V_{Q M}(g, s)\right]\right|_{\Delta g=0}=0 .
$$

$\left(\mathrm{P}_{2}\right)$ (Polynomial solution)—The solution of the quantum Hamilton-Jacobi Equation (47) takes the polynomial form (108) (harmonic representation).

$\left(\mathrm{P}_{3}\right)$ (Uniqueness property)-The 4 -scalar coefficients $a_{(o)}(s)$ and $a_{(1)}(s)$ are determined by means of ODEs which are implied by Equation (47).

$\left(\mathrm{P}_{4}\right)$ (Invariance property) - The same ODEs are identical with the corresponding equations holding in the case of stationary background field tensor (i.e., the case (3)) reported previously in Ref. [6]. Hence the 4-scalar coefficients $a_{(o)}(s)$ and $a_{(1)}(s)$ are independent of the choice of the functional setting for the background field tensor (i.e., respectively Equation (3) or Equation (2)).

Proof. To reach the thesis we introduce preliminarily a second-order, i.e., harmonic, expansion for the effective potential $V_{o}(g+\Delta g)$. Elementary algebra shows that this takes the form

$$
\begin{aligned}
& V_{o}(g+\Delta g)=V_{o}(g) \\
& +\left.\Delta g_{v}^{\mu} \frac{\partial}{\partial \Delta g_{v}^{\mu}} V_{o}(g+\Delta g)\right|_{\Delta g=0} \\
& +\left.\frac{1}{2} \Delta g_{\beta}^{\alpha} \Delta g_{v}^{\mu} \frac{\partial^{2}}{\partial \Delta g_{\beta}^{\alpha} \partial \Delta g_{v}^{\mu}} V_{o}(g+\Delta g)\right|_{\Delta g=0} .
\end{aligned}
$$


Then, substituting this expression in Equation (114), evaluation of the partial derivative gives

$$
\begin{aligned}
& \left.\frac{\partial}{\partial \Delta g_{k}^{\lambda}}\left[V_{o}(g+\Delta g)+V_{Q M}(g, s)\right]\right|_{\Delta g=0}= \\
& \left.\frac{\partial}{\partial \Delta g_{k}^{\lambda}}\left[V_{o}(g)+V_{Q M}(g, s)\right]\right|_{\Delta g=0} \\
& +\left.\frac{\partial}{\partial \Delta g_{k}^{\lambda}}\left[\Delta g_{v}^{\mu} \frac{\partial}{\partial \Delta g_{v}^{\mu}} V_{o}(g+\Delta g)\right]\right|_{\Delta g=0} \\
& +\left.\frac{\partial}{\partial \Delta g_{k}^{\lambda}}\left[\frac{1}{2} \Delta g_{\beta}^{\alpha} \Delta g_{v}^{\mu} \frac{\partial^{2}}{\partial \Delta g_{\beta}^{\alpha} \partial \Delta g_{v}^{\mu}} V_{o}(g+\Delta g)\right]\right|_{\Delta g=0} .
\end{aligned}
$$

Only the linear term in the $\Delta g_{v}^{\mu}$-expansion and the Bohm potential contribution $V_{Q M}(g, s)$ remain, so that explicit calculation recovers identically Equation (114) which is satisfied being proportional to the quantum-modified Einstein Equation (68) (in agreement with Proposition $P 2_{1}$ ). This proves $P 4_{1}$. Then one can show (Proposition $\mathrm{P}_{2}$ ) by straightforward algebra that, in analogy with Ref. [6], a polynomial solution of the quantum Hamilton-Jacobi Equation (47) exists also in the case of non-stationary background (see e.g., Equation (2)).

Next let us consider the uniqueness property (Proposition $\mathrm{P}_{3}$ ). For this purpose one needs to evaluate the covariant $s$-derivative of the quadratic terms (i.e., proportional to $\Delta g_{\alpha}^{\alpha} \Delta g_{\beta}^{\beta}$ ) in $\mathcal{S}^{(q)}\left(G_{L}(s), \Delta g, s\right)$. For this purpose one notices that upon invoking Equation (108) it follows identically that

$$
\left\{\begin{array}{c}
D_{s}\left[\Delta g_{\alpha \beta} \Delta g^{\alpha \beta}\right]=0, \\
D_{s}\left[\Delta g_{\alpha}^{\alpha} \Delta g_{\beta}^{\beta}\right]=0 .
\end{array}\right.
$$

This implies the differential identity

$$
\begin{aligned}
& D_{s}\left[\frac{a_{\alpha p}^{\beta q}(s)}{2} \Delta g_{\beta}^{\alpha} \Delta g_{q}^{p}\right]= \\
& \frac{1}{2}\left[\Delta g_{\alpha}^{\beta} \Delta g_{\beta}^{\alpha} D_{s}\left[a_{(o)}(s)\right]+\Delta g_{\alpha}^{\alpha} \Delta g_{\beta}^{\beta} D_{s}\left[a_{(1)}(s)\right]\right],
\end{aligned}
$$

where the derivatives of $a_{(o)}(s)$ and $a_{(1)}(s)$ are respectively proportional to the two 4-scalars $\Delta g_{\alpha}^{\beta} \Delta g_{\beta}^{\alpha}$ and $\Delta g_{\alpha}^{\alpha} \Delta g_{\beta}^{\beta}$, to be considered here as independent and arbitrary. Explicit evaluation of the coefficient in the quadratic term appearing in Equation (116) then shows that two distinct ODEs are determined for $a_{(o)}(s)$ and $a_{(1)}(s)$. Direct comparison with the analogous equations determined in Ref. [6] then shows that the same ODEs are independent of the specific functional setting, i.e., either Equation (2) or Equation (3). The remarkable conclusion is therefore realized by the invariance property (Proposition $\mathrm{P}_{4}$ ) of the 4-scalar coefficients $a_{(o)}(s)$ and $a_{(1)}(s)$. Q.E.D.

\subsection{GLP Gaussian Particular Solutions of the Quantum PDF}

Let us now show that also the quantum continuity Equation (46) admits, even in the case of an arbitrary non-stationary background field tensor (e.g., Equation (3)), Gaussian-like solutions of the form:

$$
\begin{aligned}
& \rho\left(G_{L}(s), \widehat{g}(s), \Delta g, r(s), s\right)= \\
& \rho\left(G_{L}\left(s_{0}\right), \widehat{g}\left(s_{0}\right), \Delta g\left(s_{0}\right), r\left(s_{o}\right), s_{o}\right) \\
& \exp \left\{-\int_{s_{0}}^{s} d s^{\prime} \frac{\partial V_{v}^{\mu}\left(G_{L}\left(s^{\prime}\right), \Delta g, r\left(s^{\prime}\right), s^{\prime}\right)}{\partial g_{L v}^{\mu}\left(s^{\prime}\right)}\right\},
\end{aligned}
$$


where, introducing the signature parameter $\theta \equiv \pm$,

$$
\begin{aligned}
& \rho\left(G_{L}\left(s_{o}\right), \Delta g\left(s_{0}\right), r\left(s_{o}\right), s_{o}\right) \equiv \\
& \frac{1}{\pi^{5} r_{t h}^{10}} \exp \left\{-\frac{\left(\Delta g\left(s_{o}\right)+\theta \widehat{g}\left(s_{o}\right)\right)^{2}}{r_{t h}^{2}}\right\} \\
\equiv & \rho_{G}\left(\Delta g\left(s_{o}\right)+\theta \widehat{g}\left(s_{o}\right)\right),
\end{aligned}
$$

identifies an initial shifted Gaussian PDF, with $\widehat{g}(s) \equiv \widehat{g}(r(s), s)$ and $\widehat{g}\left(s_{0}\right) \equiv \widehat{g}\left(r\left(s_{0}\right), s_{0}\right)$ denoting a generally non-stationary background metric tensor and its initial value at the initial proper-time $s_{0}$ evaluated along an observer's geodesic curve. In particular, in validity of the polynomial decomposition (108) for the quantum phase function $\mathcal{S}^{(q)}\left(G_{L}(s), \Delta g, r, s\right)$, the 4-scalar function $\frac{\partial V_{\mu v}\left(G_{L}\left(s^{\prime}\right), \Delta g, s^{\prime}\right)}{\partial g_{L \mu v}\left(s^{\prime}\right)}$ is found to be function of proper-time only. More precisely it takes the form

$$
\frac{\partial V_{v}^{\mu}\left(G_{L}\left(s^{\prime}\right), \Delta g, r\left(s^{\prime}\right) s^{\prime}\right)}{\partial g_{L v}^{\mu}\left(s^{\prime}\right)} \equiv 16 p^{2}\left(s^{\prime}\right) a\left(s^{\prime}\right)
$$

where $p\left(s^{\prime}\right)$ is given by Equation (A21) (see Appendix C) and the 4-scalar function $a\left(s^{\prime}\right)$ is prescribed by requiring

$$
a\left(s^{\prime}\right)=\frac{1}{2}\left[a_{(o)}\left(s^{\prime}\right)+a_{(1)}\left(s^{\prime}\right)\right]
$$

(or equivalently Equation (A19) in Appendix C). In addition, one notices that here both $r_{t h}^{2}$ and $\left(\Delta g+\theta \widehat{g}\left(s_{o}\right)\right)^{2}$ are 4-scalars and

$$
\begin{aligned}
& \left(\Delta g\left(s_{o}\right)+\theta \widehat{g}\left(s_{o}\right)\right)^{2} \equiv \\
& \left(\Delta g\left(s_{o}\right)+\theta \widehat{g}\left(s_{o}\right)\right)_{\mu \nu}\left(\Delta g\left(s_{o}\right)+\theta \widehat{g}\left(s_{o}\right)\right)^{\mu \nu},
\end{aligned}
$$

and $r_{t h}^{2}$ is a constant independent of both the 4-position $r^{\mu}$ and the proper-time $s$. In particular, one can prove also in this case that the validity of the invariance property

$$
\left(\Delta g\left(s_{0}\right)+\theta \widehat{g}\left(s_{0}\right)\right)^{2}=(\Delta g(s)+\theta \widehat{g}(s))^{2}
$$

remains preserved for arbitrary $s, s_{0} \in I$. The proof follows from elementary algebra by noting first that

$$
\begin{aligned}
\left(\Delta g\left(s_{o}\right)+\theta \widehat{g}\left(s_{o}\right)\right)^{2} \equiv & \Delta g_{v}^{\mu}\left(s_{o}\right) \Delta g_{\mu}^{\nu}\left(s_{o}\right)+4 \\
& +2 \theta \Delta g_{\mu \nu}\left(s_{o}\right) \widehat{g}^{\mu v}\left(s_{o}\right) .
\end{aligned}
$$

Indeed, thanks to Equation (85)

$$
\Delta g_{v}^{\mu}\left(s_{o}\right) \Delta g_{\mu}^{v}\left(s_{o}\right)=\Delta g_{v}^{\mu}(s) \Delta g_{\mu}^{v}(s),
$$

while for the same reason

$$
\begin{aligned}
D_{s}\left[\Delta g_{\mu v}(s) \widehat{g}^{\mu v}(s)\right] & = \\
D_{s}\left[\Delta g_{v}^{\alpha}(s) \widehat{g}_{\alpha \mu}(s) \widehat{g}^{\mu v}(s)\right] & =D_{s}\left[\Delta g_{v}^{\alpha}(s) \delta_{\alpha}^{v}\right]=0 .
\end{aligned}
$$

Hence also the equation

$$
\Delta g_{\mu v}(s) \widehat{g}^{\mu v}(s) \equiv \Delta g_{\mu \nu} \widehat{g}^{\mu v}(s)=\Delta g_{\mu \nu}\left(s_{o}\right) \widehat{g}^{\mu v}\left(s_{o}\right)
$$


necessarily holds. As a consequence one finds again as in Ref. [6] (i.e., for the case of stationary background) that

$$
\rho_{G}\left(\Delta g\left(s_{o}\right)+\theta \widehat{g}\left(s_{o}\right)\right)=\rho_{G}(\Delta g+\theta \widehat{g}(s)),
$$

which proves the statement. The conclusion is therefore that Equation (119) takes the form

$$
\begin{aligned}
& \rho\left(G_{L}(s), \Delta g, s\right)=\rho_{G}(\Delta g+\theta \widehat{g}(s)) \\
& \exp \left\{-16 \int_{s_{0}}^{s} d s^{\prime} p^{2}\left(s^{\prime}\right) a\left(s^{\prime}\right)\right\}
\end{aligned}
$$

which indeed realizes a Gaussian particular solution for the quantum PDF. Hence, the realization of the quantum PDF (119) is again independent of the choice of the functional setting of the background field tensor, respectively being prescribed either according to Equation (3) or Equation (2).

Although in principle both Gaussian solutions corresponding to $\theta=+$ and $\theta=-$ are admissible from the mathematical point of view, in the following we shall consider only the one obtained from Equation (119) by setting $\theta \equiv-$. Hence, the initial shifted Gaussian PDF dealt with in the rest of the calculations takes the form

$$
\begin{aligned}
& \rho\left(G_{L}\left(s_{o}\right), \Delta g\left(s_{o}\right), r\left(s_{o}\right), s_{o}\right) \equiv \\
& \frac{1}{\pi^{5} r_{t h}^{10}} \exp \left\{-\frac{\left(\Delta g\left(s_{o}\right)-\widehat{g}\left(s_{o}\right)\right)^{2}}{r_{t h}^{2}}\right\} \\
\equiv & \rho_{G}\left(\Delta g\left(s_{0}\right)-\widehat{g}\left(s_{o}\right)\right) .
\end{aligned}
$$

This choice has a physical basis. In fact, according to the emergent gravity picture inherent the GLP formulation of CQG-wave equation, it warrants that the GLP-quantum/stochastic expectation value of the stochastic displacement 4-tensor $\Delta g_{\mu \nu}$ recovers the correct signature of the background metric tensor, namely

$$
\left\langle\Delta g_{\mu \nu}\right\rangle=\int_{U_{g}} d(\Delta g) \rho_{G}(\Delta g-\widehat{g}(r, s)) \Delta g_{\mu \nu}=\widehat{g}_{\mu \nu}(r, s) .
$$

We refer to Ref. [6] for an exhaustive discussion of the emergent gravity phenomenon in CQG-theory and for a detailed mathematical definition of quantum expectation value, see in particular Proposition 3 and related comments in the same reference.

\subsection{Semiclassical Limit}

An important aspect of CQG-theory and the related GLP description concerns the investigation of the semiclassical limit of the quantum theory, which establishes the connection with the classical Hamiltonian structure of GR reported in Ref. [2] and provides a test of consistency of the theory itself. The study of the semiclassical limit is conveniently performed on the set of QHE, namely the quantum Hamilton-Jacobi Equation (47) and the continuity Equation (46) through its explicit analytical Gaussian solution for the quantum PDF given by Equation (130).

We consider first the quantum Hamilton-Jacobi equation, for which the semiclassical limit is prescribed letting $\hbar \rightarrow 0$. By requiring that in the same limit both $\alpha$ and $L\left(m_{0}\right)$ reduce to their classical definition and that the real limit function $\lim _{\hbar \rightarrow 0} \frac{(q)(s)}{\hbar}=\frac{\mathcal{S}(s)}{\alpha}$ exists for arbitrary $s \in I \equiv \mathbb{R}$, with $\mathcal{S}(s)$ identifying the classical reduced Hamilton principal function (see Ref. [92]), then one can shown that the quantum Hamilton-Jacobi Equation (47) reduces to the analogous classical Hamilton-Jacobi Equation (27). In fact, considering without loss of generality the case of vacuum, the semiclassical limit of Equation (47) delivers

$$
\frac{1}{\alpha} \frac{\partial \mathcal{S}(s)}{\partial s}+\frac{1}{2 \alpha^{2} L} \frac{\partial \mathcal{S}(s)}{\partial g^{\mu \nu}} \frac{\partial \mathcal{S}(s)}{\partial g_{\mu \nu}}+\lim _{\hbar \rightarrow 0} \frac{V_{Q M}(s)}{\hbar}=0
$$


where the limit $\lim _{\hbar \rightarrow 0} \frac{V_{Q M}(s)}{\hbar}=0$ holds identically. As a consequence the quantum Hamiltonian density $H^{(q)}$ necessarily must reduce to the limit function

$$
H_{R}=\frac{1}{2 \alpha L} \frac{\partial \mathcal{S}(s)}{\partial g} \frac{\partial \mathcal{S}(s)}{\partial g_{\mu \nu}} .
$$

This coincides in form with the classical normalized Hamiltonian density given above by Equation (13) in the case of vacuum, while Equation (133) reduces to the classical GR-Hamilton-Jacobi equation.

Let us now consider the semiclassical limit for the quantum continuity equation, which is investigated here by direct analysis of the analytical Gaussian solution. In this case the behavior of the free-parameter $r_{t h}^{2}$ must be prescribed when the $\operatorname{limit}_{\lim } \rightarrow 0$ holds. Here we require that

$$
r_{t h}^{2} \sim \hbar^{\gamma}
$$

with the exponent $\gamma>0$ to be later determined upon imposing that in the same semiclassical limit both the quantum Bohm potential and the quantum cosmological constant expressed in the GLP representation vanish identically. Thus, under the previous assumption the semiclassical limit on the quantum PDF is prescribed equivalently letting $\lim _{r_{t h}^{2} \rightarrow 0} \rho\left(G_{L}(s), \Delta g, s\right)$. This amounts to require the finite width of the Gaussian function to vanish in the semiclassical limit, which means that the quantum Gaussian PDF becomes a Dirac-delta function making the quantum gravitational field to "collapse" and coincide with the classical background metric tensor at initial proper-time $s_{0}$ :

$$
\lim _{r_{t h}^{2} \rightarrow 0} \rho\left(G_{L}(s), \Delta g, s\right)=\delta\left(\Delta g\left(s_{o}\right)-\widehat{g}\left(s_{o}\right)\right) .
$$

The analysis of the semiclassical limit of the QHE enables us to stress the character of the quantum modified Einstein field equations and the underlying Gaussian solution for the quantum PDF. The latter ones in fact must not be interpreted as modified classical gravitational field equations. The quantum modified Einstein equations truly include non-stationary quantum effects arising from CQG-theory, but at the same time they preserve exactly the classical form of the Einstein theory. This is made manifest by inspection of the semiclassical limit, which recovers exactly the classical equations. Thus, the present theory is not providing some type of "ad hoc" modifications of classical GR, but instead it is consistently including quantum effects computed in the framework of a covariant quantum theory of the same gravitational field.

\section{Explicit Evaluation of the Bohm Effective Potential and Source Term}

In this section the Bohm effective potential and the corresponding source term appearing in the quantum-modified Einstein tensor equation (see Equation (66)) are determined. The task is achieved based on the GLP-approach developed here in the context of the extended functional setting. In this regard it is important to acknowledge the following unique features:

- First, as shown in the present paper, the same Equation (66) has been recovered independently also in the GLP-approach, being provided in such a context by the extremal tensor Equation (114) (see THM. 4).

- Second, based on the construction of an analytic solution for the quantum PDF (see Equation (130) above) and of the corresponding quantum phase-function (the function $\mathcal{S}^{(q)}\left(G_{L}(s), \Delta g, s\right)$ determined via the polynomial representation (108)), the GLP-approach permits one to obtain also an explicit representation of the Bohm effective quantum potential (52) and corresponding source term $B_{\mu v}(s)$.

- Third, in the subsequent calculations all integrations are performed with respect to the local extremal geodesic trajectory. As a consequence the initial proper-time $s_{0}$ is set equal to $s_{0}=0$. 
In fact, based on the construction of the analytic solution for the quantum PDF indicated above (see Equation (130) in Section 7), an explicit representation of the Bohm effective quantum potential (52) follows. This is determined by a second-degree polynomial in terms of the quantum displacement field tensor $\Delta g$, namely

$$
V_{Q M}=\frac{\hbar^{2}}{4 \alpha L} \frac{8 p^{2}(s)}{r_{t h}^{2}}-\frac{\hbar^{2}}{8 \alpha L} \frac{4 p^{2}(s)}{r_{t h}^{4}}(\Delta g-\widehat{g}(s))^{2},
$$

with $\hbar$ being the reduced Planck constant, while $r_{t h}$ is still arbitrary and must be suitably determined. The rest of the notation follows from Ref. [4], with $\alpha$ being the dimensional constant defined as $\alpha=m_{0} c L$, while $m_{0}$ and $L$ are the graviton mass and $L$ its Compton length, namely $L=\frac{\hbar}{m_{0} c}$. Thanks to this result also the Bohm source tensor field $B_{\mu \nu} \equiv B_{\mu v}(s)$, prescribed by means of Equation (69), can be readily evaluated yielding the formal representation

$$
B_{\mu v}=-\frac{\hbar^{2}}{(\alpha L)^{2}} \frac{1}{r_{t h}^{4}} f(s) \widehat{g}_{\mu v}(r(s), s),
$$

with $f(s)$ being a function of proper-time defined with respect to a maximal length local geodesic trajectory. This is determined by the equation

$$
f(s) \equiv p^{3}(s)
$$

Notice that here $p(s)$ is prescribed according to Ref. [6] (see also Equation (A21) recalled in Appendix C). More precisely, it is a function of the definite integral $\int_{s_{0}}^{s} d s^{\prime} a\left(s^{\prime}\right)$ with respect to the 4-scalar function $a(s)$ (see Equation (A19)), while also requiring $s_{o}=0$ (i.e., upon identifying the local geodetics with a maximal geodesic curve). As a consequence one has that

$$
p\left(s_{0}=0\right)=1,
$$

while its precise $s$-dependence follows from the behavior of $a(s)$. Omitting here unnecessary further details on the matter we shall refer for this purpose to the related discussion already treated in the cited reference.

Determination of the CQG-Cosmological Constant $\Lambda_{C Q G}(s)$.

The representation given above (138) for the Bohm source tensor field $B_{\mu v}$ suggests its obvious connection with a suitably-prescribed notion of cosmological constant. The same tensor field $B_{\mu \nu}$ can in fact be equivalently represented as

$$
B_{\mu \nu} \equiv-\Lambda_{C Q G}(s) \widehat{g}_{\mu v}(r(s), s),
$$

with $\Lambda_{C Q G}(s)$ denoting the $C G Q$-cosmological constant

$$
\Lambda_{C Q G}(s)=\frac{\hbar^{2}}{(\alpha L)^{2}} \frac{1}{r_{t h}^{4}} f(s)
$$

and the 4-scalar function $f(s)$ is prescribed by Equation (139). A number of important features of $\Lambda_{C Q G}(s)$ emerge. First, the function $\Lambda_{C Q G}(s)$ does not depend on the 4-position $r \equiv\left\{r^{\mu}\right\}$ and hence it effectively behaves as a constant in the quantum-modified Einstein field Equation (68). A further 
feature of $\Lambda_{C Q G}(s)$ concerns its quantum origin. As a consequence one expects that $\Lambda_{C Q G}(s)$ should vanish identically in the semiclassical limit so that

$$
\lim _{\hbar \rightarrow 0} \Lambda_{C Q G}(s)=0,
$$

and, in particular, that for $\hbar \rightarrow 0$

$$
\Lambda_{\mathrm{CQG}}(s) \sim O(\hbar) .
$$

One can show that such a requirement permits us to determine consistently the still undetermined exponential factor $\gamma$ previously introduced in Equation (135). Given the analytical solution (142) the prescription of $\gamma$, in fact, follows once demanding for consistency with Equation (144) that the ratio $\frac{\hbar^{2}}{r_{t h}^{4}}$ appearing in Equation (142) is such that

$$
\frac{\hbar^{2}}{r_{t h}^{4}} \sim O(\hbar)
$$

Invoking Equation (135) this implies therefore that $\gamma=1 / 2$, namely $r_{t h} \sim \hbar^{1 / 4}$.

An equivalent representation of the CQG-cosmological constant can be achieved in terms of a suitable, strictly positive vacuum energy density

$$
\rho_{v a c}=\frac{1}{\kappa} \Lambda_{C Q G}(s) .
$$

Equation (141), in fact, can be represented equivalently via Equation (76) in terms of the corresponding quantum contribution to the stress energy tensor $\widehat{T}_{\mu \nu}^{(q)}$. This is obtained letting

$$
\widehat{T}_{\mu \nu}^{(q)} \equiv \frac{1}{\kappa} B_{\mu \nu} \equiv-\rho_{v a c} \widehat{\mathcal{g}}_{\mu v}(r, s) .
$$

Equation (147) is therefore formally analogous to that given in Ref. [76], the realization of $\rho_{v a c}$ being, however, quite different. In the present case, in fact, $\rho_{\text {vac }}$ must be identified with the graviton vacuum energy density, i.e., the vacuum energy density produced by gravitons and arising due to the Bohm interaction which acts on the same gravitons.

Finally, a notable feature of $\Lambda_{C Q G}(s)$ concerns its proper-time dependence occurring through the strictly positive 4-scalar function $f(s)$ (see Equation (139)). In view of the prescription of the function $p(s)$ (see Equation (A18) in Appendix C) it follows that its initial value is $p\left(s_{o}\right)=1$ also for $s_{o}=0$. Hence the initial value of $\Lambda_{C Q G}(s)$ occurring at $s=s_{o}=0$ is

$$
\Lambda_{C Q G}\left(s_{0}\right)=\frac{\hbar^{2}}{(\alpha L)^{2}} \frac{1}{r_{t h}^{4}}
$$

so that the relationship between $\Lambda_{C Q G}(s)$ and $\Lambda_{C Q G}\left(s_{o}\right)$ is simply

$$
\Lambda_{C Q G}(s)=\Lambda_{C Q G}\left(s_{0}\right) p^{3}(s) .
$$

The issue over the prescription of the proper-time dependence of $\Lambda_{C Q G}(s)$ and the related analysis of qualitative properties is addressed in the next section.

\section{Proper-Time Behavior of $\Lambda_{C Q G}(s)$ and Physical Implications}

In view of Equations (139) and (A21) (see Appendix C) the prescription of the proper-time functions $f(s)$ and $p(s)$ requires in turn the evaluation of the 4-scalar function $a(s)$ given by Equation (A19). As discussed above the same function is realized by the Equation (122), with $a_{(o)}(s)$ and $a_{(1)}(s)$ being the 4-scalar coefficients appearing in the quadratic term of Harmonic representation of the quantum-phase function (108). According to Ref. [6] the same functions are determined by an 
initial-value problem associated with appropriate first-order ODEs. In the present case, one notices that $\Lambda$ must be identified with the CQG-cosmological constant $\Lambda_{\mathrm{CQG}}(s)$ so that Equations (148) and (149) must be taken into account. One obtains accordingly for $a_{(o)}(s)$ and $a_{(1)}(s)$ the two equations:

$$
\left\{\begin{aligned}
\frac{1}{4} \frac{d}{d s} a_{(o)}(s) & =\frac{p^{2}(s)}{8 \alpha L} a_{(o)}^{2}(s)+\frac{\alpha L}{2} \Lambda\left(s_{o}\right) p^{2}(s)+ \\
- & \frac{\alpha L}{2} \Lambda\left(s_{o}\right) p^{3}(s)+G_{(o)} \\
\frac{1}{4} \frac{d}{d s} a_{(1)}(s)= & \frac{p^{2}(s)}{8 \alpha L}\left(4 a_{(1)}^{2}(s)+2 a_{(o)}(s) a_{(1)}(s)\right)+ \\
- & \frac{\alpha L}{2} \Lambda\left(s_{o}\right) p^{3}(s)+G_{(1)},
\end{aligned}\right.
$$

where $G_{(o)}$ and $G_{(1)}$ are arbitrary constant gauge functions which can be conveniently chosen in such a way that the same equations admit a stationary solution. When cast in dimensionless form upon letting

$$
\left\{\begin{array}{c}
\theta=\frac{2 s}{L} \\
\bar{a}_{(o)}(\theta)=\frac{a_{(o)}(\theta)}{\alpha} \\
\bar{a}_{(1)}(\theta)=\frac{a_{(1)}}{\alpha} \\
\bar{\Lambda}_{o}=\Lambda\left(s_{o}\right) L^{2}
\end{array}\right.
$$

these yield

$$
\left\{\begin{array}{c}
\frac{d}{d \theta} \bar{a}_{(o)}(\theta)=\frac{p^{2}(\theta)}{4} \bar{a}_{(o)}^{2}(\theta)-\frac{\bar{a}_{(o)}^{2}}{4}\left(\theta_{o}\right)+\bar{\Lambda}\left(s_{o}\right)\left[p^{2}(\theta)-1\right]- \\
-\bar{\Lambda}\left(s_{o}\right)\left[p^{3}(\theta)-1\right], \\
\frac{d}{d \theta} \bar{a}_{(1)}(\theta)=\frac{p^{2}(\theta)}{4}\left(4 \bar{a}_{(1)}^{2}(\theta)+2 \bar{a}_{(o)}(\theta) \bar{a}_{(1)}(\theta)\right)+ \\
-\bar{\Lambda}\left(s_{o}\right)\left[p^{3}(\theta)-1\right]-\frac{3}{4} \bar{a}_{(1)}^{2}\left(\theta_{o}\right)+\frac{\bar{a}_{(o)}^{2}}{4}\left(\theta_{o}\right) .
\end{array}\right.
$$

Then, by setting the initial conditions

$$
\left\{\begin{array}{l}
a_{(o)}\left(\theta_{0}\right)=\widehat{a}_{(o)}, \\
a_{(1)}\left(\theta_{0}\right)=\widehat{a}_{(1)},
\end{array}\right.
$$

with $\left(\widehat{a}_{(o)}, \widehat{a}_{(1)}\right)$ being initial constants, and by requiring also

$$
\widehat{a}_{(1)}=-\widehat{a}_{(o)}
$$

it follows that Equation (152) admit the stationary solution $\bar{a}_{(o)}(\theta) \equiv \widehat{a}_{(o)}, \bar{a}_{(1)}(\theta) \equiv-\widehat{a}_{(o)}$ and $\bar{p}(\theta) \equiv 1$.

We now pose the problem of the investigation of the asymptotic property of the solutions of Equations (152) and (153), i.e., for solutions such that

$$
\left\{\begin{array}{c}
\lim _{\theta \rightarrow \infty} \bar{a}(\theta)=0 \\
\lim _{\theta \rightarrow \infty} \bar{a}_{(o)}(\theta)=-\lim _{\theta \rightarrow \infty} \bar{a}_{(1)}(\theta)=\bar{a}_{(o) \infty} \\
\lim _{\theta \rightarrow \infty} \frac{d}{d \theta} \bar{a}_{(o)}(\theta)=\lim _{\theta \rightarrow \infty} \frac{d}{d \theta} \bar{a}_{(1)}(\theta)=0 \\
\lim _{\theta \rightarrow \infty} p(\theta)=p_{\infty} \\
\lim _{\theta \rightarrow \infty} \bar{\Lambda}(s)=\bar{\Lambda}_{C Q G \infty} .
\end{array}\right.
$$

In this regard the following result holds. 
Theorem 5 (Asymptotic behavior of the solutions of Equation (152)). Assuming that Equation (155) and the limits $\bar{a}_{(o) \infty}$ and $p_{\infty}$ exist, with $\bar{a}_{(o) \infty}$ and $p_{\infty}$ being non-vanishing, then the following propositions apply: $\left(P 5_{1}\right)$ First, the equation

$$
p_{\infty}^{2}=\frac{1+\frac{1}{4} \frac{\bar{a}_{(1)}^{2}\left(\theta_{o}\right)}{\bar{\Lambda}\left(s_{o}\right)}-\frac{1}{2} \frac{\bar{a}_{(o)}^{2}\left(\theta_{o}\right)}{\bar{\Lambda}\left(s_{o}\right)}}{1-\frac{1}{4} \frac{\bar{a}_{(o) \infty}^{2}}{\bar{\Lambda}\left(s_{o}\right)}}
$$

holds.

$\left(P 5_{2}\right)$ Second, depending whether $p_{\infty}^{2}>1$ or $p_{\infty}^{2}<1$, it follows respectively that

$$
\Lambda_{C Q G \infty}>\Lambda_{C Q G}\left(s_{o}\right),
$$

or

$$
\Lambda_{C Q G \infty}<\Lambda_{C Q G}\left(s_{0}\right) .
$$

In particular, in validity of the initial conditions (154) it follows that

$$
\Lambda_{C Q G \infty}=\Lambda_{C Q G}\left(s_{0}\right) .
$$

Proof. The proof of proposition $P 5_{1}$ follows from elementary algebra. Indeed, let us evaluate the limits for $s \rightarrow+\infty$ of Equation (152). In validity of Equation (155) these become respectively

$$
\left\{\begin{array}{c}
0=\frac{p_{\infty}^{2}}{4} \bar{a}_{(o) \infty}^{2}-\frac{\bar{a}_{(o)}^{2}}{4}\left(\theta_{o}\right)+\bar{\Lambda}\left(s_{o}\right)\left[p_{\infty}^{2}-1\right]- \\
-\bar{\Lambda}\left(s_{o}\right)\left[p_{\infty}^{3}-1\right], \\
0=\frac{p_{\infty}^{2}}{4}\left(4 \bar{a}_{(1) \infty}^{2}+2 \bar{a}_{(o) \infty}(\theta) \bar{a}_{(1) \infty}(\theta)\right)+ \\
-\bar{\Lambda}\left(s_{0}\right)\left[p_{\infty}^{3}-1\right]-\frac{3}{4} \bar{a}_{(1)}^{2}\left(\theta_{0}\right)+\frac{1}{4} \bar{a}_{(o)}^{2}\left(\theta_{o}\right) .
\end{array}\right.
$$

Subtracting the second equation from the first one it then follows

$$
p_{\infty}^{2}=\frac{\frac{1}{2} \bar{a}_{(1)}^{2}\left(\theta_{0}\right)-\frac{1}{4} \bar{a}_{(o)}^{2}\left(\theta_{o}\right)-\bar{\Lambda}\left(s_{o}\right)}{\frac{1}{4} \bar{a}_{(o) \infty}^{2}-\bar{\Lambda}\left(s_{0}\right)},
$$

which implies Equation (156). Similarly, the inequalities Equations (157)-(159) are immediate consequences of Equations (139) and (142). Q.E.D.

THM. 5 yields sufficient conditions for the establishment of the asymptotic behavior of the CQG-cosmological constant. It follows, depending on the initial conditions, that the asymptotic value of the cosmological constant $\Lambda_{C Q G \infty}$ can in principle be either larger or smaller than the initial value $\Lambda_{C Q G}\left(s_{0}\right)$ provided that the asymptotic limit $\bar{a}_{(o) \infty}^{2} \equiv \bar{a}_{(1) \infty}^{2}$ is suitably well behaved. However, the issue remains under which initial conditions (53) $p_{\infty}^{2}$ can be respectively $>1$ or $<1$.

To answer this question let us consider for definiteness the case of small amplitude solutions corresponding to initial conditions of the type

$$
\left\{\begin{array}{c}
a_{(o)}\left(\theta_{o}\right)=\widehat{a}_{(o)}+\Delta a_{(o)}, \\
a_{(1)}\left(\theta_{o}\right)=-\widehat{a}_{(o)}+\Delta a_{(1)}, \\
\left|\frac{\Delta a_{(o)}}{\widehat{a}_{(o)}}\right|,\left|\frac{\Delta a_{(1)}}{\widehat{a}_{(o)}}\right| \ll 1
\end{array}\right.
$$


namely of the form

$$
\left\{\begin{array}{c}
p_{\infty}=1+\Delta p_{\infty}, \\
a_{(o) \infty}=\widehat{a}_{(o)}+\Delta a_{(o) \infty \prime} \\
a_{(1) \infty}=-\widehat{a}_{(o)}-\Delta a_{(o) \infty}, \\
\left|\Delta p_{\infty}\right|,\left|\frac{\Delta a_{(o) \infty}}{\widehat{a}_{(o)}}\right| \ll 1 .
\end{array}\right.
$$

Then elementary algebra shows from Equation (150) that

$$
\Delta p_{\infty} \cong-\frac{2 \widehat{a}_{(o)} \Delta a_{(1)}}{3 \bar{\Lambda}\left(\theta_{o}\right)\left(1-\frac{5}{24} \frac{\widehat{a}_{(o)}^{2}}{\Lambda\left(\theta_{o}\right)}\right)} .
$$

This implies, therefore, that depending on the initial conditions (see in particular Equation (162)) $\Delta p_{\infty}$ in principle can indeed be either positive or negative and hence $p_{\infty}$ respectively $>1$ or $<1$.

\section{Physical Implications}

Given the results established so far, physical implications and qualitative properties of the resulting quantum-modified Einstein field equations can be established. The main feature arising from the discussion presented above is that it provides a generally non-stationary realization for $\Lambda_{C Q G}(s)$, leading in turn to a corresponding non-stationary background space-time of the form (2). In the case of vacuum considered here this means that the latter may be identified, for example, with a non-stationary de Sitter space-time $\left\{\mathbf{Q}^{4}, \widehat{g}(r, s)\right\}$, i.e., an expanding universe having an explicitly proper-time dependent cosmological constant

$$
\Lambda(s)=\Lambda_{\text {bare }}+\Lambda_{C Q G}(s),
$$

as the only source of curvature, with the explicit proper-time dependence contained in $\Lambda_{C Q G}(s)$ being prescribed according to CQG-theory via second-quantization effects. The equation which determines the cosmological constant (165), however, still contains arbitrary free parameters (see also Equation (142)), i.e., besides the (possible) classical contribution $\Lambda_{b a r e}$ also $r_{t h}$. One notices, in particular, that $\Lambda_{\text {bare }}$ remains in principle completely undetermined at this stage. Indeed no account has been given here for a classical physical mechanism that can possibly justify a non-vanishing contribution of this kind. For this reason, ruling out possible classical modifications of Einstein field equation, its contribution can be ignored in the present framework, thus yielding the identification

$$
\Lambda(s) \equiv \Lambda_{C Q G}(s)
$$

As a consequence, upon introducing the function $B(s) \equiv\left(1-\frac{r^{2}}{A(s)^{2}}\right)$, with $A(s)$ identifying the de Sitter characteristic length, the Riemann distance in the de Sitter space-time $\left\{\mathbf{Q}^{4}, \widehat{g}(r, s)\right\}$ when expressed in spherical coordinates $(c t, r, \vartheta, \varphi)$ takes the form:

$$
\begin{aligned}
d s^{2} & =B(s) c^{2} d t^{2}-B(s)^{-1} d r^{2}+r^{2} d \Omega^{2} \\
& \equiv \widehat{g}_{\mu \nu}(r, s) d r^{\mu} d r^{\nu} .
\end{aligned}
$$

Therefore the corresponding space-time background metric tensor becomes

$$
\widehat{g}_{\mu v}(r, s)=\operatorname{diag}\left\{B(s), B(s)^{-1}, r^{2}, r^{2} \sin ^{2} \vartheta\right\} .
$$


Here the parameter $A \equiv A(s)$ is related to $\Lambda(s)$ by means of the prescription

$$
A(s)=\sqrt{\frac{3}{\Lambda(s)}} .
$$

Hence this means that in turn $d s^{2}$ necessarily must depend on the maximal geodesic curve on which the tangent infinitesimal displacement $d r^{\mu} \equiv d r^{\mu}(s)$ is evaluated. One notices, in particular, that the same parameter must be suitably associated with the radius of the de Sitter event horizon, i.e., the region of space-time which can be reached only by particles which, after starting from the Big Bang event, have traveled at the speed of light. As such they are necessarily realized by photons whose world-lines are null geodesic trajectories. As a consequence, for these trajectories it must be that $s=s_{0} \equiv 0$ so that both $A\left(s_{o}=0\right)$ and $\Lambda\left(s_{O}=0\right)$ are necessarily identified with pure constants. In particular $\Lambda\left(s_{o}=0\right)$ can be identified with the radius of the de Sitter event horizon, i.e., prescribed so that $B\left(s_{0}=0\right)=0$.

Regarding the physical identification of the initial value $A\left(s_{0}=0\right) \equiv \sqrt{\frac{3}{\Lambda\left(s_{0}=0\right)}}$, this would require in turn knowledge of the precise value of $\Lambda\left(s_{0}=0\right)$. However, a possible order-of-magnitude estimate can be achieved assuming that the initial value of the cosmological constant was comparable to its current experimental value, namely

$$
\Lambda_{o b s} \cong 1.2 \times 10^{-52} m^{-2} .
$$

Thus, upon letting for example that

$$
\Lambda\left(s_{o}=0\right) \sim \Lambda_{o b s}
$$

this yields for $A\left(s_{o}=0\right)$ the estimate

$$
A\left(s_{o}=0\right) \sim \sqrt{\frac{3}{\Lambda_{o b s}}} \sim 10^{10} l y .
$$

As a consequence the initial value $A\left(s_{o}=0\right)$ is comparable, in order of magnitude, with the theoretical estimate of the current radius of the universe, namely

$$
\lambda_{t h} \cong 1.38 \times 10^{10} l y
$$

It must be stressed, however, that the precise estimate of $A\left(s_{0}=0\right)$ is also subject to the validity of Equation (166), namely it can be modified if additional (classical/quantum) contributions to the cosmological constant are included in the theory. Regarding, instead, the still undetermined quantum 4-scalar and dimensionless parameter $r_{t h}$ a numerical estimate can be obtained as follows. First, thanks to the prescriptions for $\alpha$ and $L$ recalled above (see Ref. [4]), the ratio $\frac{\hbar^{2}}{(\alpha L)^{2}}$ becomes $\frac{\hbar^{2}}{(\alpha L)^{2}}=\frac{1}{L^{2}}$, with $L$ denoting again the graviton Compton length defined above. Next, in validity of Equation (166), let us now require for definiteness that $\Lambda(s)$ coincides in order of magnitude with $\Lambda_{o b s}$. In this case adopting for the graviton mass the theoretical estimate given in Ref. [4] one finds that $r_{t h}^{2} \cong 0.326$. As a consequence the Gaussian quantum PDF (130) remains prescribed, with its half-way amplitude (namely $r_{t h}^{2}$ ) being necessarily of $O(1)$, i.e., safely in the quantum regime (in fact validity of the semiclassical regime would require instead $r_{t h}^{2} \rightarrow 0$ ). In other words, the same PDF has a finite "thermal spread", so that it exhibits an intrinsic quantum character. Furthermore, the same result warrants also validity of the Equation (166), a choice which is consistent with the graviton mass estimate given in Ref. [4].

Equation (166) is the main result of the paper. The physical implications of the CQG-prediction of the cosmological constant are potentially wide-range. The main one, besides the identification of the non-stationary de Sitter space-time, concerns the physical interpretation of the quantum origin of the cosmological constant. Unlike earlier conjectures that the quantum contribution to the cosmological 
constant should be ascribed to quantum-vacuum energy density arising from all possible quantum fields [74], CQG-theory shows that $\Lambda_{Q M} \equiv \Lambda_{C Q G}(s)$ is actually produced by the Bohm interaction only due to a quantum vacuum populated by gravitons only, i.e., without requiring any additional quantum or classical field. More precisely, as shown by Equation (69), the cosmological constant behaves generally as a non-stationary (with respect to the invariant proper-time s) field generated by the gravitational field itself through quantum self interaction. However, it follows clearly that it is not the vacuum energy density per se which is responsible for a non-vanishing cosmological constant, but rather the fluctuations of its probability density. From the mathematical point of view this is expressed by the fact that the quantum cosmological constant term entering Equations (68) is generated specifically due to the gradient of the Bohm potential, which here has the physical meaning of a vacuum gravitational quantum interaction. In addition, it can be concluded that this same mechanism of generation of $\Lambda_{Q M}$ is also consistent with the existence of quantum massive gravitons, as it follows from the results previously reported in Ref. [4] where the inclusion of a cosmological constant in the quantum wave equation was shown to generate a discrete invariant-energy spectrum for the same massive gravitons characteristic of manifestly-covariant quantum gravity theory.

Further interesting implications concern the comparison with the large-scale structure of the universe and in particular evidences \#1-\#5 (see Section 1.1 in the Introduction). We stress that for this purpose a systematic, i.e., detailed numerical, analysis of the solution (167) or equivalent (168) (both to be considered in validity of Equation (166)) is required, being left to future work. Nevertheless its consistency with the large-scale structure of the universe can still be formally established as discussed above. Let us briefly outline some of the interesting conclusions that emerge in this way.

Consider, in particular, evidence \#1 about the flatness property of the de Sitter space-time. Indeed, this is characterized by a Ricci curvature 4-scalar $R=R(s)$ such that $R(s)=4 \Lambda(s)$. If one assumes the ordering estimate $\Lambda\left(s_{0}=0\right) \sim \Lambda(s)$ it follows, consistent with such a property, that $R(s) \ll 1$. Similarly, evidence \#2 (the lack of large-scale correlations among "distant" regions of universe) is direct consequence of spherical symmetry property of the de Sitter metric tensor. The third evidence about the isotropic and uniform character of the expansion/acceleration of the universe at large distances is again consistent with the same symmetry property and the strict positivity of the quantum cosmological constant determined here. Furthermore, regarding the "Big Bang hypothesis" (see evidence \#4), as discussed in Section 2, this is already built-in in CQG-theory itself. Finally, concerning the issue of the inflationary transient phase of the early universe (evidence \#5 ), we conclude that its possible existence-based on the inequality estimates given above—cannot be ruled out.

Finally, a comment must be made on the possible implications for cosmology and actual physical relevance related to the determination of the CQG-cosmological constant and specifically to the prediction of its possible proper-time dependence achieved here. This refers to the issue whether the theory presented here may be adequate in a quantitative sense to explain the large-scale phenomenology of the universe. The same issue is particularly relevant for the possible theoretical prediction and suggested explanation of the observed values of the expansion rate and acceleration of the universe. It seems wise to state that at this stage definite conclusions are still premature. Indeed, before drawing definite conclusions, a systematic analysis of the proper-time dependence predicted here for the cosmological constant as well a deeper analysis of the same phenomenology are required. Nevertheless, the fact is that the prediction of the proper-time behavior determined by the CQG-cosmological constant appears, at least in qualitative sense, compatible or in agreement both with the possible existence of an inflationary stage in the early universe and the tentative suggested explanation of the phenomena of expansion and acceleration of the universe based on CQG-theory. According to CQG-theory, in fact, such phenomena should arise due to the quantum self-generation of the cosmological constant, namely the vacuum quantum Bohm interaction occurring among massive gravitons. As a consequence no additional external sources or classical/quantum 
interactions are actually needed to determine the proper-time dependence and observed value of the cosmological constant.

This conclusion departs from previous literature in at least three respects. The first one is the physical origin of the cosmological constant. In previous literature in fact, by far the most popular conjecture is usually regarded to be the vacuum energy density $\left(\rho_{A}\right)$ associated with dark matter/energy as the possible physical cause able to explain both the expansion and acceleration of the universe as well as the cosmological constant $[47,84,85]$. The second one, is that former theoretical approaches are phenomenological in character and do not provide a self-consistent theory for constructing either the quantum-modified Einstein field equations or the energy density $\rho_{A}$ itself. Third and final, there is no obvious connection between the same quantum-modified Einstein field equations (which are manifestly covariant) and previous quantum theories of gravity (which typically are not so).

\section{Concluding Remarks}

In this paper key issues have been address which are related to the determination of the (quantum) cosmological constant in the context of manifestly-covariant quantum gravity (CQG-theory). These have included in particular:

1. The definition of the observer's proper-time $(s)$, consistent with the treatment adopted in CQG-theory of gravitons as classical point-particles and with the Big Bang hypothesis. This is prescribed as the arc length of a suitable non-null geodesic world-line associated with the background metric tensor $\widehat{g}$, which represents a virtual trajectory, namely one of the infinite possible physically admissible worldlines, associated with a massive graviton. To this end the same curve is identified in a cosmological framework with an observer's maximal geodetics, i.e., a geodesic curve having the maximal arc length and with origin point $r^{\mu}\left(s_{0}\right)$, the point of creation of the same particle, coinciding with (or suitably close to) the Big Bang event. By construction for the initial 4-position $r^{\mu}\left(s_{o}\right)$ is therefore such that $r^{\mu}\left(s_{0}\right) \equiv r^{\mu}\left(s_{o}=0\right)$.

2. The establishment of the Hamiltonian structure of CQG-theory. This is represented by a set of continuous canonical equations (referred to here as quantum Hamilton equations) whose validity is implied by the quantum-wave equation through its corresponding quantum Hamilton-Jacobi equation. As shown in THM. 1, the same Hamiltonian structure remains preserved also in validity of the said extended setting (i.e., for non-stationary background metric tensor).

3. The discovery of quantum-modified Einstein field equation. In fact, the quantum Hamilton equations have been shown to admit a particular realization in terms of a set of PDEs which is analogous to the classical Einstein Equation (5) but in which quantum source terms are taken into account. Remarkably also such an equation remains preserved under the same extended functional setting (see THM. 2).

4. The establishment of the corresponding formulation of the generalized Lagrangian path (GLP) approach. The issues indicated above have been cast in the framework provided by the said, earlier formulated, GLP-approach. The key feature of the GLP-approach unveiled here (THM. 3 and THM. 4) concerns its validity also in the context of the extended functional setting and the determination of explicit vacuum solutions of the quantum hydrodynamic equations associated with the CQG-wave equation, with particular reference to quantum solutions characterized by Gaussian quantum PDFs.

5. The prescription of the quantum cosmological constant, its estimate achieved in the framework of CQG-theory and its dynamical behavior. In fact it has been shown that the cosmological constant $\Lambda \equiv \Lambda_{C Q G}(s)$ is non-stationary, i.e., dependent on the observer's proper-time $s$. The determination of the proper-time dependence of the quantum cosmological constant has been based on the GLP-approach which permits the construction of dynamically-consistent analytic solutions for 
the quantum wave-function. As a result the relevant asymptotic properties (for $s \rightarrow \infty$ ) of the $s$-dependent quantum cosmological constant have been established (THM. 5).

6. The implications and possible interpretation of the large-scale phenomenology of the universe by means of an extended formulation of CQG-theory in which the background space-time itself is non-stationary. For this purpose the associated background metric field tensor $\widehat{g} \equiv\left\{\widehat{g}_{\mu v}\right\}$ has been couched in an extended functional setting in which the same tensor field is considered of the form $\widehat{g}(r, s) \equiv\left\{\widehat{g}_{\mu v}(r, s)\right\}$, namely again explicitly dependent on the same proper-time $s$.

The conclusions are relevant at least for two main reasons.

The first one refers to a peculiar emergent-gravity feature, previously referred to (see Ref. [6]) as "first-type emergent-gravity paradigm", according to which the Einstein field equations themselves should be implied by quantum theory of SF-GR. As a consequence this means that in such a context also the precise form of the background space-time, i.e., the background field tensor itself should be determined in terms of a suitable particular solution of the quantum-wave equation appropriate for the same quantum theory. Such a feature, in our view, can be regarded as a true test of consistency for arbitrary quantum theories of gravity. Indeed the ultimate goal of any theory of this type should be the prediction of the background metric tensor of the universe and its corresponding tensor field equation which in the context of SF-GR coincides with the Einstein field equations. In this paper such a property has been shown to hold for CQG-theory based on the quantum-modified Einstein field equations indicated above. The remarkable feature is, in fact, that as shown by THM. 2 these are achieved without performing any limiting approximation, such as the semiclassical continuum limit which is obtained letting $\hbar \rightarrow 0$ in the quantum-wave equation.

However, in this connection, two additional side-consequences follow. The first one is a fundamental physical restriction. Indeed, due to the manifest-covariance property of the Einstein field equations a stringent condition arises also on the class of possible, i.e., physically-admissible, quantum field theories of gravity. In fact, it is obvious that the same ones should necessarily be restricted to manifestly-covariant ones, namely realized, as CQG-theory, by means of a 4-tensor field theory endowed with tensor properties prescribed with respect to the same background field tensor $\widehat{g}$. The second side-implication concerns the physical interpretation and role of CQG-theory. In fact it cannot merely be viewed as one of the possible background space-time theories, i.e., in which the background field tensor is arbitrarily prescribed as a particular solution of the classical Einstein field equations. On the contrary it must be intended as a truly self-consistent quantum theory of gravity in which the background field tensor $\widehat{g}$ is a solution of the corresponding quantum-modified Einstein field equations, namely in which the cosmological constant is, in turn, uniquely prescribed by means of CQG-theory itself.

The further aspect worth to be mentioned concerns the explicit prescription and properties of the background space-time arising in the context of CQG-theory. This is identified here with the non-stationary de Sitter space-time $\left\{\mathbf{Q}^{4}, \widehat{g}(r, s)\right\}$, being $\widehat{g}(r, s)$ the corresponding metric field-tensor characterized by the non-stationary cosmological constant $\Lambda \equiv \Lambda_{C Q G}(s)$. In particular, remarkable features which emerge in this connection are that: (A) The prediction of the initial value $\Lambda_{C Q G}\left(s_{o}=0\right)$ obtained here is consistent with the graviton mass estimate established in a previous paper in the case (see Ref. [4]). (B) The unique second-quantization character of the cosmological constant, which arises due to the Bohm interaction and more precisely due to the gradient of the Bohm effective quantum potential. (C) The universal property of the cosmological constant, i.e., the fact that its existence is independent of the possible presence of additional external fields and further classical or quantum interactions.

These conclusions provide also further theoretical insight on the axiomatic foundations as well as physical implications of CQG-theory for large-scale phenomena of the universe. In the present investigation crucially-important second-quantization aspects of the theory have been studied. These concern the possible explanation, in such a context, of the physical mechanism responsible for the occurrence of the cosmological constant, as well the possible existence of an inflationary phenomenon 
in the early universe, and, in turn, also a suggested physical explanation for the closely-related observed values of the expansion rate and acceleration of the universe.

Nevertheless, CQG-theory is still built upon a first-quantization approach (realized by the so-called g-quantization) which fulfills the quantum unitarity principle and, consequently, the conservation of quantum probability associated with the quantum wave function. As such, no trans-Planckian effects, nor possible information losses arising at event horizons in black-hole space-times are taken into account in the current formulation of CQG-theory. Nevertheless, the inclusion of additional second-quantization effects is in principle possible, with particular reference to quantum modifications of the background space-time at the Planck length, such as the inclusion of localized quantum particle sources.In view of these considerations, CQG-theory may be expected to provide fertile grounds for new conceptual developments and a variety of applications in quantum gravity and quantum cosmology.

Author Contributions: As a part of a long-standing collaboration between the two authors, this paper was started during the Summer of 2017. The ideas expressed here are fruit of careful joint discussions between the authors. The initial motivation of the work is related to the investigation of CQG-theory (C. Cremaschini and $\mathrm{M}$. Tessarotto, 2017) and the related formulation of the GLP-approach developed in the same context (M. Tessarotto and C. Cremaschini, 2018).

Funding: Investigation developed within the research project of the Albert Einstein Center for Gravitation and Astrophysics, Czech Science Foundation No. 14-37086G (M.T.). The authors acknowledge institutional support by the Silesian University in Opava, Czech Republic.

Acknowledgments: This work is dedicated to the dearest memory of Flavia, wife of M.T. and motherly friend of C.C., recently passed away. The authors are grateful to the International Center for Theoretical Physics (ICTP Trieste, Italy) for the hospitality during the initial preparation of the manuscript.

Conflicts of Interest: The authors declare no conflict of interest.

\section{Appendix A. Classical Kinetic and Normalized Effective Potential Densities}

The effective kinetic and the normalized effective potential density $T_{R}$ and $V$ appearing in the classical Hamiltonian density $H_{R}(13)$ take the form (see Refs. [3,4])

$$
\left\{\begin{array}{c}
T_{R} \equiv \frac{1}{2 \alpha L f(h)} \pi_{\mu \nu} \pi^{\mu \nu,} \\
V(g, \widehat{g}, r, s) \equiv \sigma V_{o}(g, \widehat{g}, r, s)+\sigma V_{F}(g, \widehat{g}, r, s),
\end{array}\right.
$$

with $h$ being the variational weight-factor

$$
h(g, \widehat{g}(r, s))=2-\frac{1}{4} g^{\alpha \beta} g^{\mu v} \widehat{g}_{\alpha \mu}(r, s) \widehat{g}_{\beta v}(r, s),
$$

while $L$ and $\alpha$ are constants, i.e., suitable 4 -scalars both identified according to the treatment given in Ref. [4]. In addition, $V$ and $V_{F}$ represent respectively the vacuum and external field contributions (see definitions in Ref. [3]),

$$
\begin{aligned}
& V_{o} \equiv h \alpha L\left[g^{\mu v} \widehat{R}_{\mu v}-2 \Lambda\right], \\
& V_{F}(g, \widehat{g}, r) \equiv h L_{F}(g, \widehat{g}, r),
\end{aligned}
$$

where $\widehat{R}_{\mu \nu} \equiv R_{\mu \nu}(\widehat{g})$ and $\Lambda$ identify respectively the background Ricci tensor and the cosmological constant, $L_{F}$ being associated with a non-vanishing stress-energy tensor, while $f(h)$ and $\sigma$ denote suitable multiplicative gauge functions identified with $f(h)=1$ and $\sigma=-1$. 


\section{Appendix B. Covariant Partial Derivative}

In the case of non-stationary background metric tensor (see Equation (3)) the operator $\left.\frac{d}{d s}\right|_{r}$ appearing in Equation (18) must be prescribed in such a way to satisfy the covariance property (4) also for the covariant and countervariant components of the tensor field

$$
\left\{\begin{aligned}
H_{\mu v} & \left.\equiv \frac{d}{d s}\right|_{r} x_{\mu v} \\
H^{\mu v} & \left.\equiv \frac{d}{d s}\right|_{r} x^{\mu \nu}
\end{aligned}\right.
$$

with $x_{\mu v}$ denoting the canonical 4-tensors $x_{\mu v}=g_{\mu v}, \pi_{\mu v}$ for which by construction

$$
\left\{\begin{array}{l}
x^{p q} \widehat{g}_{\mu^{\prime}} \widehat{g}_{v^{\prime} q}=x_{\mu^{\prime} v^{\prime}} \\
x_{p q} \widehat{g}^{\mu^{\prime}} \widehat{g}^{v^{\prime} q}=x^{\mu^{\prime} v^{\prime}} .
\end{array}\right.
$$

For definiteness, denoting in the following $\widehat{g}_{\mu \alpha}=\widehat{g}_{\mu \alpha}(r, s)$ and $\widehat{g}^{\mu \beta}=\widehat{g}^{\mu \beta}(r, s)$, the identities $\left.\left.\frac{d}{d s}\right|_{s} \widehat{g}_{\mu \alpha} \equiv \frac{d}{d s}\right|_{s} \widehat{g}^{\mu \beta} \equiv 0$ hold. Furthermore, noting that the non-stationary background metric tensor must satisfy as well the orthogonality condition

$$
\widehat{g}_{\mu \alpha} \widehat{g}^{\mu \beta}=\delta_{\alpha}^{\beta}
$$

it follows that also the identity

$$
\widehat{g}^{\mu \beta} \frac{\partial}{\partial s} \widehat{g}_{\mu \alpha}+\widehat{g}_{\mu \alpha} \frac{\partial}{\partial s} \widehat{g}^{\mu \beta}=0
$$

must hold. Then one can prove that, thanks to Equations (A5)-(A7), the following prescriptions for the covariant partial derivatives $\left.\frac{d}{d s}\right|_{r}$ hold:

$$
\left\{\begin{array}{l}
\left.\frac{d}{d s}\right|_{r} x^{\alpha \beta}=\frac{\partial}{\partial s} x^{\alpha \beta}-\frac{1}{2} x^{p q} \widehat{g}_{\mu^{\prime}} \widehat{g}_{v^{\prime}} \frac{\partial}{\partial s}\left(\widehat{g}^{\alpha \mu^{\prime}} \widehat{g}^{\beta \nu^{\prime}}\right), \\
\left.\frac{d}{d s}\right|_{r} x_{\alpha \beta}=\frac{\partial}{\partial s} x_{\alpha \beta}-\frac{1}{2} x_{p q} \widehat{g}^{\mu^{\prime}} p_{\widehat{g}^{\prime}}{ }^{\nu^{\prime}} \frac{\partial}{\partial s}\left(\widehat{g}_{\alpha \mu^{\prime}} \widehat{g}_{\beta v^{\prime}}\right) .
\end{array}\right.
$$

In view of the orthogonality conditions (A6) these can be equivalently written as

$$
\left\{\begin{array}{l}
\left.\frac{d}{d s}\right|_{r} x^{\alpha \beta}=\frac{\partial}{\partial s} x^{\alpha \beta}-\frac{1}{2} x_{\mu^{\prime} v^{\prime}} \frac{\partial}{\partial s}\left(\widehat{g}^{\alpha \mu^{\prime}} \widehat{g}^{\beta v^{\prime}}\right), \\
\left.\frac{d}{d s}\right|_{r} x_{\alpha \beta}=\frac{\partial}{\partial s} x_{\alpha \beta}-\frac{1}{2} x^{\mu^{\prime} v^{\prime}} \frac{\partial}{\partial s}\left(\widehat{g}_{\alpha \mu^{\prime}} \widehat{g}_{\beta v^{\prime}}\right) .
\end{array}\right.
$$

To prove Equation (A8) one notices in fact that

$$
\begin{aligned}
& \left.\widehat{g}_{\mu \alpha} \widehat{g}_{\nu \beta} \frac{d}{d s}\right|_{r} x^{\alpha \beta}=\widehat{g}_{\mu \alpha} \widehat{g}_{\nu \beta} \frac{\partial}{\partial s} x^{\alpha \beta} \\
& -\widehat{g}_{\mu \alpha} \widehat{g}_{\nu \beta} \frac{1}{2} x^{p q} \widehat{g}_{\mu^{\prime}} \widehat{g}_{v^{\prime} q} \frac{\partial}{\partial s}\left(\widehat{g}^{\alpha \mu^{\prime}} \widehat{g}^{\beta \nu^{\prime}}\right),
\end{aligned}
$$

where

$$
\widehat{g}_{\mu \alpha} \widehat{\delta}_{\nu \beta} \frac{\partial}{\partial s} x^{\alpha \beta}=\frac{\partial}{\partial s} x_{\mu v}-x^{\alpha \beta} \frac{\partial}{\partial s}\left(\widehat{g}_{\mu \alpha} \widehat{g}_{\nu \beta}\right)
$$


and

$$
\begin{aligned}
& \widehat{g}_{\mu \alpha} \widehat{g}_{v \beta} \frac{1}{2} x^{p q} \widehat{g}_{\mu^{\prime}} \widehat{g}_{v^{\prime} q} \frac{\partial}{\partial s}\left(\widehat{g}^{\alpha \mu^{\prime}} \hat{g}^{\beta v^{\prime}}\right)= \\
& -\widehat{g}^{\alpha \mu^{\prime}} \widehat{g}^{\beta v^{\prime}} \frac{1}{2} x^{p q} \widehat{g}_{\mu^{\prime}} \widehat{\widehat{g}}_{v^{\prime} q} \frac{\partial}{\partial s}\left(\widehat{g}_{\mu \alpha} \widehat{g}_{\nu \beta}\right)= \\
& -\frac{1}{2} x^{\alpha \beta} \frac{\partial}{\partial s}\left(\widehat{g}_{\mu \alpha} \widehat{g}_{\nu \beta}\right) .
\end{aligned}
$$

As a consequence, elementary algebra yields

$$
\begin{aligned}
\left.\widehat{g}_{\mu \alpha} \widehat{\delta}_{\nu \beta} \frac{d}{d s}\right|_{r} x^{\alpha \beta} & =\frac{\partial}{\partial s} x_{\mu \nu}-\frac{1}{2} x^{\alpha \beta} \frac{\partial}{\partial s}\left(\widehat{g}_{\mu \alpha} \widehat{g}_{\nu \beta}\right) \equiv \\
& \left.\equiv \frac{d}{d s}\right|_{r} x_{\mu \nu},
\end{aligned}
$$

and similarly

$$
\left.\widehat{g}^{\mu \alpha} \widehat{g}^{\nu \beta} \frac{d}{d s}\right|_{r} x_{\alpha \beta}=\left.\frac{d}{d s}\right|_{r} x^{\mu \nu}
$$

Furthermore, as a consequence of the prescriptions (A8) (or equivalent of Equation (A9)), it is immediate to prove the distributive property holds

$$
D_{s}\left(x^{\alpha \beta} x_{\alpha \beta}\right)=x^{\alpha \beta} \frac{d}{d s} x_{\alpha \beta}+x_{\alpha \beta} \frac{d}{d s} x^{\alpha \beta},
$$

with $D_{s}$ and $\frac{d}{d s}$ being respectively the covariant $s$-derivatives (21) and (18). This proves the validity of the covariance property for the 4-tensor field defined by Equation (A4) and hence of the prescriptions (A8) for the covariant partial derivatives $\left.\frac{d}{d s}\right|_{r}$.

\section{Appendix C. Determination of the 4-Scalar Factor $p(s)$}

In analogy to Ref. [6] let us introduce for the quantum phase-function $\mathcal{S}^{(q)}\left(G_{L}(s), \Delta g, s\right)$ the "harmonic" polynomial decomposition realized in terms of a second-degree polynomial of the form (104). Then, in analogy to Ref. [6] the determination of the 4-scalar factor $p(s)$ is provided by the following propositions (with proofs analogous to those given in Appendix B of Ref. [6]).

Proposition A1 (Determination of the tensor field $\left.\frac{\partial \Delta g_{\beta}^{\alpha}}{\partial \delta g_{L v}^{\mu}\left(s^{\prime}\right)}\right)$. Given validity of the polynomial representation (104), the tensor field $\frac{\partial \Delta g_{\beta}^{\alpha}}{\partial \delta_{L v}^{\mu}\left(s^{\prime}\right)}$ takes the form

$$
\frac{\partial \Delta g_{\beta}^{\alpha}}{\partial \delta g_{L v}^{\mu}\left(s^{\prime}\right)}=-\frac{\partial \Delta g_{\beta}^{\alpha}}{\partial G_{L v}^{\mu}\left(s^{\prime}\right)},
$$

with

$$
\frac{\partial \Delta g_{\beta}^{\alpha}}{\partial \delta g_{L v}^{\mu}\left(s^{\prime}\right)}=\delta_{\mu}^{\alpha} \delta_{\beta}^{v} p(s),
$$

and $p(s)$ being the 4 -scalar function determined by the integral equation

$$
p(s)=\frac{1}{1+\int_{s_{0}}^{s} d s^{\prime} \frac{1}{\alpha L} a\left(s^{\prime}\right) p\left(s^{\prime}\right)} .
$$

Here $a(s)$ is the 4-scalar function

$$
a(s) \equiv \frac{1}{4} a_{q \beta}^{p \alpha}(s) \delta_{p}^{q} \delta_{\alpha}^{\beta},
$$


with $a_{q \beta}^{p \alpha}(s)$ being the tensor introduced in the polynomial decomposition of the phase function $\mathcal{S}^{(q)}$ given above by Equation (104).

Proposition A2 (Determination of the 4-scalar function $p(s)$ ). In validity of Equation (A18) it follows that

$$
|p(s)|=\frac{1}{\left(1+\frac{2}{\alpha L} \int_{s_{0}}^{s} d s^{\prime} a\left(s^{\prime}\right)\right)^{1 / 2}}
$$

which upon requiring $p\left(s_{0}\right)=1$ delivers

$$
p(s)=\frac{1}{\left(1+\frac{2}{\alpha L} \int_{s_{0}}^{s} d s^{\prime} a\left(s^{\prime}\right)\right)^{1 / 2}} .
$$

\section{References}

1. Cremaschini, C.; Tessarotto, M. Synchronous Lagrangian variational principles in General Relativity. Eur. Phys. J. Plus 2015, 130, 123. [CrossRef]

2. Cremaschini, C.; Tessarotto, M. Manifest covariant Hamiltonian theory of General Relativity. Appl. Phys. Res. 2016, 8, 2. [CrossRef]

3. Cremaschini, C.; Tessarotto, M. Hamiltonian approach to GR-Part 1: Covariant theory of classical gravity. Eur. Phys. J. C 2017, 77, 329. [CrossRef]

4. Cremaschini, C.; Tessarotto, M. Hamiltonian approach to GR-Part 2: Covariant theory of quantum gravity. Eur. Phys. J. C 2017, 77, 330. [CrossRef]

5. Cremaschini, C.; Tessarotto, M. Quantum-wave equation and Heisenberg inequalities of covariant quantum gravity. Entropy 2017, 19, 339. [CrossRef]

6. Tessarotto, M.; Cremaschini, C. Generalized Lagrangian path approach to manifestly-covariant quantum gravity theory. Entropy 2018, 20, 205. [CrossRef]

7. Escofet, A.; Elizalde, E. Gauss-Bonnet modified gravity models with bouncing behavior. Mod. Phys. Lett. A 2016, 31, 1650108. [CrossRef]

8. Elizalde, E.; Odintsov, S.V.; Sebastiani, L.; Myrzakulov, R. Beyond-one-loop quantum gravity action yielding both inflation and late-time acceleration. Nucl. Phys. B 2017, 921, 411-435. [CrossRef]

9. Messiah, A. Quantum Mechanics; Dover Pubs: New York, NY, USA, 1999.

10. Einstein, A. The Meaning of Relativity; Princeton University Press: Princeton, NJ, USA, 2004.

11. Landau, L.D.; Lifschitz, E.M. Field Theory, Theoretical Physics; Addison-Wesley: New York, NY, USA, 1957; Volume 2.

12. Misner, C.W.; Thorne, K.S.; Wheeler, J.A. Gravitation, 1st ed.; W.H. Freeman and Company: New York, NY, USA, 1973.

13. Tessarotto, M.; Cremaschini, C. Theory of Nonlocal Point Transformations in General Relativity. Adv. Math. Phys. 2016, 2016, 1-32, doi:10.1155/2016/9619326. [CrossRef]

14. Jordi, G.; Narciso, R. Multisymplectic unified formalism for Einstein-Hilbert gravity. J. Math. Phys. 2018, $59,032502$.

15. Tessarotto, M.; Cremaschini, C. Generalized Lagrangian-path representation of non-relativistic quantum mechanics. Found. Phys. 2016, 46, 1022-1061. [CrossRef]

16. Mortonson, M.J. Testing flatness of the universe with probes of cosmic distances and growth. Phys. Rev. D 2009, 80, 123504. [CrossRef]

17. Okouma, P.M.; Fantaye, Y.; Bassett, B.A. How flat is our Universe really? Phys. Lett. B 2013, 719, 1-4. [CrossRef]

18. Pawłowski, T.; Pierini, R.; Wilson-Ewing, E. Loop quantum cosmology of a radiation-dominated flat FLRW universe. Phys. Rev. D 2014, 90, 123538. [CrossRef] 
19. Fathi, M.; Jalalzadeh, S.; Moniz, P.V. Classical universe emerging from quantum cosmology without horizon and flatness problems. Eur. Phys. J. C 2016, 76, 527. [CrossRef]

20. Weinberg, S. The Cosmological Constant Problem. Rev. Mod. Phys. 1989, 16, 1-23. [CrossRef]

21. Carroll, S. Spacetime and Geometry; Addison Wesley: San Francisco, CA, USA, 2004; pp. 171-174.

22. Pawłowski, T.; Ashtekar, A. Positive cosmological constant in loop quantum cosmology. Phys. Rev. D 2012, 85, 064001. [CrossRef]

23. Holland, J.; Hollands, S. A small cosmological constant due to non-perturbative quantum effects. Class. Quant. Grav. 2014, 31, 125006. [CrossRef]

24. Szydłowski, M. Cosmological model with decaying vacuum energy from quantum mechanics. Phys. Rev. D 2015, 91, 123538. [CrossRef]

25. Oda, I. Quantum aspects of nonlocal approach to the cosmological constant problem. Phys. Rev. D 2017, 96, 024027. [CrossRef]

26. Szulc, Ł. An open FRW model in loop quantum cosmology. Class. Quant. Grav. 2007, 24, 6191. [CrossRef]

27. Brizuela, D.; Marugan, G.A.M.; Pawłowski, T. Fast Track Communication: Big Bounce and inhomogeneities. Class. Quant. Grav. 2010, 27, 052001. [CrossRef]

28. Sadjadi, H.M. On solutions of loop quantum cosmology. Eur. Phys. J. C 2013, 73, 2571. [CrossRef]

29. Cai, Y.-F.; Wilson-Ewing, E. Non-singular bounce scenarios in loop quantum cosmology and the effective field description. J. Cosmol. Astropart. Phys. 2014, 3, 026. [CrossRef]

30. Agullo, I. Loop quantum cosmology, non-Gaussianity, and CMB power asymmetry. Phys. Rev. D 2015, 92, 064038. [CrossRef]

31. Oikonomou, V.K. Inflation and bounce from classical and loop quantum cosmology imperfect fluids. Int. J. Mod. Phys. D 2017, 26, 1750110. [CrossRef]

32. Alesci, E.; Botta, G.; Cianfrani, F.; Liberati, S. Cosmological singularity resolution from quantum gravity: The emergent-bouncing universe. Phys. Rev. D 2017, 96, 046008. [CrossRef]

33. Li, B.-F.; Singh, P.; Wang, A. Towards cosmological dynamics from loop quantum gravity. Phys. Rev. D 2018, 97, 084029. [CrossRef]

34. Misner, C.W. The Isotropy of the Universe. Astrophys. J. 1968, 151, 431. [CrossRef]

35. Gamow, G. Observational Properties of the Homogeneous and Isotropic Expanding Universe. Phys. Rev. Lett. 1968, 20, 1310. [CrossRef]

36. Collins, C.B.; Hawking, S.W. Why is the Universe Isotropic? Astrophys. J. 1973, 180, 317. [CrossRef]

37. Hawking, S.W.; Luttrell, J.C. The isotropy of the universe. Phys. Lett. B 1984, 143, 83-86. [CrossRef]

38. Anninos, P.; Matzner, R.A.; Rothman, T.; Ryan, M.P., Jr. How does inflation isotropize the Universe? Phys. Rev. D 1991, 43, 3821. [CrossRef]

39. Barrow, J.D.; Kodama, H. The isotropy of compact universes. Class. Quant. Grav. 2001, 18, 1753. [CrossRef]

40. Räsänen, S. Relation between the isotropy of the CMB and the geometry of the universe. Phys. Rev. D 2009, 79, 123522. [CrossRef]

41. Saadeh, D.; Feeney, S.M.; Pontzen, A.; Peiris, H.V.; McEwen, J.D. How Isotropic is the Universe? Phys. Rev. Lett. 2016, 117, 131302. [CrossRef] [PubMed]

42. Ǩípa, J.; Shafieloo, A. Testing the Isotropic Universe Using the Gamma-Ray Burst Data of Fermi/GBM. Astrophys. J. 2017, 851, 15. [CrossRef]

43. Wang, Y.; Wang, F.Y. Testing the isotropy of the Universe with Type Ia supernovae in a model-independent way. Mon. Not. R. Astron. Soc. 2018, 474, 3516-3522.

44. Pinto-Neto, N.; Santini, E.S. The accelerated expansion of the Universe as a quantum cosmological effect. Phys. Lett. A 2003, 315, 36-50. [CrossRef]

45. Schmidt, B.P. Nobel Lecture: Accelerating expansion of the Universe through observations of distant supernovae. Rev. Mod. Phys. 2012, 84, 1151. [CrossRef]

46. Haridasu, B.S.; Luković, V.V.; D’Agostino, R.; Vittorio, N. Strong evidence for an accelerating Universe. Astron. Astrophys. 2017, 600, L1. [CrossRef]

47. Riess, A.G. Nobel Lecture: My path to the accelerating Universe. Rev. Mod. Phys. 2012, 84, 1165. [CrossRef]

48. Susskind, L. The Cosmic Landscape: String Theory and the Illusion of Intelligent Design; Little, Brown and Company: New York, NY, USA, 2005.

49. Nussbaumer, H. The Discovery of the Expanding Universe and 80 Years of Big Bang. Int. J. Mod. Phys. D 2011, 20, 87. [CrossRef] 
50. Rebhan, E. Cosmic inflation and big bang interpreted as explosions. Phys. Rev. D 2012, 86, 123012. [CrossRef]

51. Penrose, R. Singularities in big-bang cosmology. Q. J. R. Astron. Soc. 1988, 29, 61-63.

52. Battisti, M.V.; Montani, G. The Big-Bang singularity in the framework of a Generalized Uncertainty Principle. Phys. Lett. B 2007, 656, 96-101. [CrossRef]

53. Koslowski, T.A.; Mercati, F.; Sloan, D. Through the big bang: Continuing Einstein's equations beyond a cosmological singularity. Phys. Lett. B 2018, 778, 339-343. [CrossRef]

54. Ashtekar, A.; Barrau, A. Some Conceptual Issues in Loop Quantum Cosmology. Class. Quant. Grav. 2015, 32, 234001. [CrossRef]

55. Benetti, M.; Landau, S.J.; Alcaniz, J.S. Constraining quantum collapse inflationary models with CMB data. J. Cosmol. Astropart. Phys. 2016, 12, 035. [CrossRef]

56. León, G. Eternal inflation and the quantum birth of cosmic structure. Eur. Phys. J. C 2017, 77, 705. [CrossRef]

57. Brandenberger, R. Initial conditions for inflation-A short review. Int. J. Mod. Phys. D 2017, 26, 1740002. [CrossRef]

58. Szydłowski, M.; Stachowski, A. Simple cosmological model with inflation and late times acceleration. Eur. Phys. J. C 2018, 78, 249. [CrossRef]

59. Guth, A.H. Inflationary universe: A possible solution to the horizon and flatness problems. Phys. Rev. D 1981, 23, 347-356. [CrossRef]

60. Ade, P.A.R.; Aghanim, N.; Armitage-Caplan, C.; Arnaud, M.; Ashdown, M.; Aumont, J.; Baccigalupi, C.; Banday, A.J.; Barreiro, R.B.; Bartlett, J.G.; et al. Planck 2015 results. XIII. Cosmological parameters. Astron. Astrophys. 2016, 594, A13.

61. Einstein, A. Kosmologische Betrachtungen zur allgemeinen Relativitätstheorie (Cosmological Considerations in the General Theory of Relativity); Sitzungsberichte; Koniglich Preußische Akademie der Wissenschaften: Berlin, Germany, 1917; pp. 142-152.

62. Ivanov, A.N.; Wellenzohn, M. Standard electroweak interactions in gravitational theory with chameleon field and torsion. Phys. Rev. D 2015, 91, 085025. [CrossRef]

63. Ivanov, A.N.; Wellenzohn, M. Einstein-Cartan Gravity with Torsion Field Serving as an Origin for the Cosmological Constant or Dark Energy Density. Astrophys. J. 2016, 829, 47. [CrossRef]

64. Azri, H.; Bounames, A. Geometrical origin of the cosmological constant. Gen. Relativ. Gravit. 2012, 44, 2547-2561. [CrossRef]

65. Lu, J.; Ma, L.; Liu, M.; Wu, Y. Time variable cosmological constant of holographic origin with interaction in Brans-Dicke theory. Int. J. Mod. Phys. D 2012, 21, 1250005. [CrossRef]

66. Dymnikova, I. The cosmological term as a source of mass. Class. Quant. Grav. 2002, 19, 725. [CrossRef]

67. Dymnikova, I. From vacuum nonsingular black hole to variable cosmological constant. Grav. Cosmol. Suppl. 2002, 8N1, 131.

68. Bronnikov, K.A.; Dobosz, A.; Dymnikova, I.G. Nonsingular vacuum cosmologies with a variable cosmological term. Class. Quant. Grav. 2003, 20, 3797. [CrossRef]

69. Majerník, V. Letter: A Cosmological Constant Interpreted as the Field Energy of a Quaternionic Field. Gen. Relativ. Gravit. 2003, 35, 1833. [CrossRef]

70. Azri, H.; Bounames, A. Cosmological Consequences of a Variable Cosmological Constant Model. Int. J. Mod. Phys. D 2017, 26, 1750060. [CrossRef]

71. Rugh, S.; Zinkernagel, H. The quantum vacuum and the cosmological constant problem. Stud. Hist. Philos. Mod. Phys. 2001, 33, 663-705. [CrossRef]

72. Wang, Q.; Zhu, Z.; Unruh, W. How the huge energy of quantum vacuum gravitates to drive the slow accelerating expansion of the Universe. Phys. Rev. D 2017, 95, 103504. [CrossRef]

73. Cree, S.S.; Davis, T.M.; Ralph, T.C.; Wang, Q.; Zhu, Z.; Unruh, W.G. Can the fluctuations of the quantum vacuum solve the cosmological constant problem? arXiv 2018, arXiv:1805.12293v1.

74. Garriga, J.; Vilenkin, A. Solutions to the Cosmological Constant Problems. Phys. Rev. D 2001, 64, 023517. [CrossRef]

75. Crittenden, R.; Majerotto, E.; Piazza, F. Measuring Deviations from a Cosmological Constant: A Field-Space Parametrization. Phys. Rev. Lett. 2007, 98, 251301. [CrossRef] [PubMed]

76. Barrow, J.D.; Shaw, D.J. New solution of the cosmological constant problems. Phys. Rev. Lett. 2011, 106, 101302. [CrossRef] [PubMed]

77. Cicciarella, F.; Pieroni, M. Universality for quintessence. J. Cosmol. Astropart. Phys. 2017, 8, 010. [CrossRef] 
78. Asenjo, F.A.; Hojman, S.A. Class of Exact Solutions for a Cosmological Model of Unified Gravitational and Quintessence Fields. Found. Phys. 2017, 47, 887-896. [CrossRef]

79. Bianchi, E.; Krajewski, T.; Rovelli, C.; Vidotto, F. Cosmological constant in spinfoam cosmology. Phys. Rev. D 2011, 83, 104015. [CrossRef]

80. Garattini, R.; Nicolini, P. Noncommutative approach to the cosmological constant problem. Phys. Rev. D 2011, 83, 064021. [CrossRef]

81. Ali, A.F.; Das, S. Cosmology from quantum potential. Phys. Lett. B 2014, 741, 276-279.

82. Das, S. Quantum Raychaudhuri equation. Phys. Rev. D 2014, 89, 084068. [CrossRef]

83. Lashin, E.I. On the correctness of cosmology from quantum potential. Mod. Phys. Lett. A 2016, $31,07$. [CrossRef]

84. Perlmutter, S.; Aldering, G.; Goldhaber, G.; Knop, R.A.; Nugent, P.; Castro, P.G.; Deustua, S.; Fabbro, S.; Goobar, A.; Groom, D.E.; et al. Measurements of Omega and Lambda from 42 High-Redshift Supernovae. Astron. J. 1999, 517, 565. [CrossRef]

85. Peebles, P.J.E.; Ratra, B. The cosmological constant and dark energy. Rev. Mod. Phys. 2003, 75, 559. [CrossRef]

86. Han, M. Einstein equation from covariant loop quantum gravity in semiclassical continuum limit. Phys. Rev. D 2017, 96, 024047. [CrossRef]

87. Arnowitt, R.; Deser, S.; Misner, C.W. Gravitation: An Introduction to Current Research; Witten, L., Ed.; Wiley: New York, NY, USA, 1962.

88. Etienne, Z.B.; Liu, Y.T.; Shapiro, S.L. Relativistic magnetohydrodynamics in dynamical spacetimes: A new AMR implementation. Phys. Rev. D 2010, 82, 084031. [CrossRef]

89. Alcubierre, M. Introduction to 3+1 Numerical Relativity; Oxford University Press: Oxford, UK, 2008.

90. Gheorghiu, T.; Vacaru, O.; Vacaru, S. Off-diagonal deformations of kerr black holes in Einstein and modified massive gravity and higher dimensions. Eur. Phys. J. C 2014, 74, 3152. [CrossRef]

91. Ruchin, V.; Vacaru, O.; Vacaru, S. On relativistic generalization of Perelman's W-entropy and thermodynamic description of gravitational fields and cosmology. Eur. Phys. J. C 2017, 77, 184. [CrossRef]

92. Cremaschini, C.; Tessarotto, M. Exact solution of the EM radiation-reaction problem for classical finite-size and Lorentzian charged particles. Eur. Phys. J. Plus 2011, 126, 42. [CrossRef]

93. Cremaschini, C.; Tessarotto, M. Hamiltonian formulation for the classical EM radiation-reaction problem: Application to the kinetic theory for relativistic collisionless plasmas. Eur. Phys. J. Plus 2011, 126, 63. [CrossRef]

94. Wald, R. General Relativity; University of Chicago Press: Chicago, IL, USA, 1984.

95. Rovelli, C. Space and Time in Loop Quantum Gravity. In Beyond Spacetime: The Philosophical Foundations of Quantum Gravity; Biha, B.L., Matsubara, K., Wuthrich, C., Eds.; Cambridge University Press: Cambridge, UK, 2018.

96. Bohm, D.; Hiley, B.J.; Kaloyerou, P.N. An ontological basis for the quantum theory. Phys. Rep. 1987, 144, 321-375. [CrossRef]

97. Grössing, G. On the thermodynamic origin of the quantum potential. Phys. A Stat. Mech. Its Appl. 2009, 388, 811-823. [CrossRef]

98. Dennis, G.; de Gosson, M.A.; Hiley, B.J. Bohm's quantum potential as an internal energy. Phys. Lett. A 2015, 379, 1224-1227. [CrossRef]

99. Tessarotto, M.; Mond, M.; Batic, D. Hamiltonian Structure of the Schrödinger Classical Dynamical System. Found. Phys. 2016, 46, 1127-1167. [CrossRef]

100. DeWitt, B.S. Quantum Theory of Gravity. I. The Canonical Theory. Phys. Rev. 1967, 160, 1113. [CrossRef]

(c) 2018 by the authors. Licensee MDPI, Basel, Switzerland. This article is an open access article distributed under the terms and conditions of the Creative Commons Attribution (CC BY) license (http:// creativecommons.org/licenses/by/4.0/). 